\title{
Tidal downscaling from the open ocean to the coast: a new approach applied to the Bay of Biscay
}

\author{
Toublanc F. ${ }^{1,}{ }^{*}$, Ayoub N. K. ${ }^{1}$, Lyard F. ${ }^{1}$, Marsaleix P. ${ }^{2}$, Allain D. J. ${ }^{1}$
}

1 Univ Toulouse, CNRS, LEGOS, CNES,IRD,UPS, F-31400 Toulouse, France.

2 Univ Toulouse, CNRS, LA, F-31400 Toulouse, France.

* Corresponding author : F. Toublanc, email address : florence.toublanc@legos.obs-mip.fr

\begin{abstract}
:
Downscaling physical processes from a large scale to a regional scale 3D model is a recurrent issue in coastal processes studies. The choice of boundary conditions will often greatly influence the solution within the 3D circulation model. In some regions, tides play a key role in coastal dynamics and must be accurately represented.

The Bay of Biscay is one of these regions, with highly energetic tides influencing coastal circulation and river plume dynamics. In this study, three strategies are tested to force with barotropic tides a 3D circulation model with a variable horizontal resolution. The tidal forcings, as well as the tidal elevations and currents resulting from the 3D simulations, are compared to tidal harmonics extracted from satellite altimetry and tidal gauges, and tidal currents harmonics obtained from ADCP data.

The results show a strong improvement of the M2 solution within the 3D model with a "tailored" tidal forcing generated on the same grid and bathymetry as the 3D configuration, compared to a global tidal atlas forcing. Tidal harmonics obtained from satellite altimetry data are particularly valuable to assess the performance of each simulation. Comparisons between sea surface height time series, a sea surface salinity database, and daily averaged $2 \mathrm{D}$ currents also show a better agreement with this tailored forcing.
\end{abstract}

\section{Highlights}

- A new approach is proposed to tidal downscaling in 3D coastal models. The best tidal solution is not necessarily the best forcing for downscaling. The error on the M2 elevation in the Bay of Biscay is reduced by $75 \%$. The success of the method is also assessed through its impact on circulation. Satellite altimetry is a powerful tool for tidal assessment.

Keywords : Tides, Downscaling, 3D coastal modelling, Boundary conditions, Satellite altimetry, Bay of Biscay 
Please note that this is an author-produced PDF of an article accepted for publication following peer review. The definitive publisher-authenticated version is available on the publisher Web site. 


\section{Introduction}

2 Increasing efforts are made to improve the accuracy of global circulation models at regional scales, by improving 3 the grid resolution, by taking into account more physical processes or through data assimilation techniques (e.g. Holt 4 et al. (2017)). In spite of significant progresses in the recent years, the global or basin simulations performance 5 generally remains insufficient to accurately study coastal phenomena, and regional models are still the best option, 6 thanks to their higher resolution, tuned parameterizations or parameters, and to the consideration of comprehensive 7 coastal processes such as tides, surface waves, estuarine processes, etc. Since regional physical processes are partly 8 driven by large scale processes (Zheng and Weisberg, 2012), with this limited-area approach comes the issue of

\footnotetext{
${ }^{*}$ Corresponding author

Email address: florence. toublanc@legos .obs-mip.fr (F. Toublanc)
} 
downscaling and managing open boundary conditions. As first stated by Oliger and Sundström (1978), open boundary conditions can never be considered as perfect. Several strategies have been developed to deal with this issue, as discussed for instance by Blayo and Debreu (2005) and Herzfeld (2009). More particularly, the nesting of several grids within each other are often used to gradually increase the resolution near the coast. However, the interpolation necessary due to resolution differences and bathymetry inconsistencies may induce errors at the open boundaries.

Modelling the 3D ocean circulation in coastal areas and shelf seas requires an accurate representation of the tidal dynamics, especially near the coast. The tidal solution in a regional circulation model results from the introduction of the astronomical tidal potential in the primitive equations, and from open boundary conditions in sea surface elevation (hereafter SSH) and currents. The accuracy of the tidal forcing at the open boundaries is critical for the representation of tides of course, but also for the simulation of mixing and circulation through different mechanisms: non-linear interactions between tidal currents and the general circulation, mixing induced by internal tides, bottom friction modulation by tidal currents, mixing enhancing by vertical tidal currents shear (Carter and Merrifield, 2007; Herzfeld, 2009; Guarnieri et al., 2013). Guarnieri et al. (2013) show the impact of tides on the Adriatic Sea circulation, with a 3D model. They find that tides influence the circulation by modifying the horizontal advection, especially during periods of weak wind stress. They also assess the impact of tides on mixing, this time for strong wind stress periods. Residual tidal flows due to non-linear interactions with the topography ('topographic rectification') can also be generated (González-Pola et al., 2012; Wang et al., 2013). Holt et al. (2017) show that the inclusion of tides in circulation models allows a better representation of seasonal stratification cycles than high resolution models without tides.

In principle, the tidal forcing at the open-boundaries is given either as a set of tidal constituents or as time-varying fields of sea surface elevation and horizontal currents. The latter option is for instance tempting when the coastal model is also forced at the open-boundaries by a large-scale circulation model that simulates both the tidal and nontidal circulation. However, such an option requires the availability of the large-scale forcing at very high-frequency (a few minutes) which is, in practice, never (or very rarely) possible. That is why in most cases, the open-boundary conditions for tides and for the non-tidal circulation are prescribed as distinct sources. We have adopted such an approach in the present study.

Downscaling tides in a coastal (child) model is not a trivial issue: as for the general problem of open-boundary conditions, the difficulties come from the numerical scheme or from the prescribed fields (at last for incoming conditions or 'active boundaries') stemming from the parent model. Another difficulty is introduced if the model is also forced at the open boundaries (hereafter OB) by low frequency motions. For instance, Herzfeld and Gillibrand (2015) discuss the problem of dealing with multiple timescales in a scheme based on local adjustment of the flux at the OB; they propose an approach based on dual relaxation timescales for their scheme. In general, the use of prescribed tidal fields lead to inconsistencies with the interior solution, mainly due to differences in bathymetry between the forcing and forced models. As an example, Wang et al. (2013) note that an adjustment of the prescribed tidal barotropic velocity at the $\mathrm{OB}$ is necessary to ensure consistency of the depth integrated barotropic transport with the interior 
solution. In the case of baroclinic tides, other complications come from possible inconsistencies between the child and parent stratification as well as from the non-stationary part of the internal wave fields that requires the availability of the parent outputs at very high-frequency. For these reasons, in cases of offline downscaling problems such as the one addressed in this paper, only the barotropic tides are taken into account.

To prescribe barotropic tides at the $\mathrm{OB}$, two strategies are usually adopted. The most common one is based on the use of tidal atlases that provide tidal harmonics (amplitude and phase) of sea surface elevation and, in most case, of barotropic velocities for a given tidal spectrum. Several global atlases exist and are regularly updated (for a review see Stammer et al. (2014)): some of them are built from empirical adjustment mostly from satellite altimetry to a prior model, such as the GOT (Ray, 1999), or TPXO (Egbert and Erofeeva, 2002) models. Other atlases are solutions of barotropic hydrodynamical models constrained by satellite and/or in situ observations via data assimilation. Among the latter, FES2012 is the last distributed product from a long series of solutions obtained with the T-UGOm hydrodynamical model (Lyard et al., 2006) described in section 2.2. (At the time when we write this paper, the FES2014 atlas is under construction). Several examples of regional or coastal circulation models that prescribe tidal harmonics from global atlases at their open-boundaries are found in the recent literature: Dong et al. (2011) and Wang et al. (2013) use the TPXO.6 solution for their regional models in the Southern California Bight and Prince William Sound respectively; Katavouta and Thompson (2016) use FES2004 over the Nova Scotia Shelf. In coastal/estuarine applications, one or several levels of nesting are often necessary and the open-boundary conditions may be obtained from a larger scale model, as done in Toublanc et al. (2016).

Another strategy consists in running the regional or coastal model in a 2D mode without any other forcing than tidal harmonics in sea surface elevation at the $\mathrm{OB}$ (the latter provided by an atlas). The solution of this barotropic simulation gives tidal constituents that are then used to force the model in 3D mode. The tidal spectrum that can be estimated from the 2D run depends mainly on the length of the simulation. Such an approach has been used in the North-East Atlantic by Maraldi et al. (2013).

In this paper, we therefore address the issue of downscaling barotropic tides in a circulation model, where an accurate representation of tides is required either for the tidal signal itself (both barotropic tides and internal tides) or for its impact on the circulation and hydrology. There is a wide literature on open-boundary conditions (hereafter OBC) in regional models, and many variants of the Dirichlet, Flather, radiation and relaxation conditions are developed, based on different implementations on the model grid and different strategies regarding sponge layers. A thorough work with the SYMPHONIE model has been made to implement relevant OBC for coastal applications in presence of strong or weak tides and consideration to fundamental properties (such as conservation of mass, energy) has been given. This is summarized in the paper of Marsaleix et al. (2006). We have not found any drawbacks with this scheme. We do not claim its superiority to alternative schemes either.

The two main sources of errors arising with $\mathrm{OBC}$ are the errors linked to the equations and numerical implementation of the $\mathrm{OBC}$ method and those due to the possible inconsistency between external forcing and interior dynamics; in this study we have made the choice to address the latter only. This is a choice motivated by the need to find a 
relatively easy and fast-to-implement method, that can be applied in different configurations, as an alternative to the revisit or adjustment of the numerical scheme and equations.

Our objective in this paper is to propose a robust and simple approach that allows to improve the downscaling of barotropic tides for any given set of boundary equations and of external forcing, therefore being non-intrusive in the model equations. In other words, given a certain 3D circulation model, with a given grid and bathymetry, how can we improve the tidal forcing to reduce errors on the interior tidal solution? Our new approach is based on the additional use of a tidal model, here the T-UGOm model of Lyard et al. (2006). Our 3D coastal model is SYMPHONIE (Marsaleix et al., 2008, 2009). To avoid inconsistencies between the prescribed tides and the interior solution due to mesh resolution and bathymetry differences, tidal boundary conditions are generated on the same grid and bathymetry as the ones used by the 3D circulation model. The unstructured 2D spectral model T-UGOm was adapted to perform simulations on a structured, variable horizontal resolution grid, by introducing C-grid equivalent quadrangle elements.

This approach is applied to the Bay of Biscay, where tides are highly energetic, particularly over the western French shelf with tidal ranges reaching $6 \mathrm{~m}$ locally at the coast. Tides are dominated by M2 (Cavanie and Hyacinthe, 1976; Cartwright et al., 1980; Le Cann, 1990), with amplitudes ranging between 1 to 2 m, against a few centimeters for K1. Non-linear interactions occurring between semi-diurnal constituents and the topography can result in the generation of overtides such as M4, which can reach amplitudes of $25 \mathrm{~cm}$. Le Cann (1990) showed that the width of the Bay of Biscay is close to resonance for quarter-diurnal tides, leading to a strong amplification of these constituents. Figure 1 shows the distribution of the M2 tide (elevation and current) in the Bay of Biscay, taken from the FES2012 tidal atlas. In addition, Table 1 gives the minimum, mean and maximum values for the tidal amplitude of M2, S2, M4 and $\mathrm{K} 1$, in the Bay of Biscay.

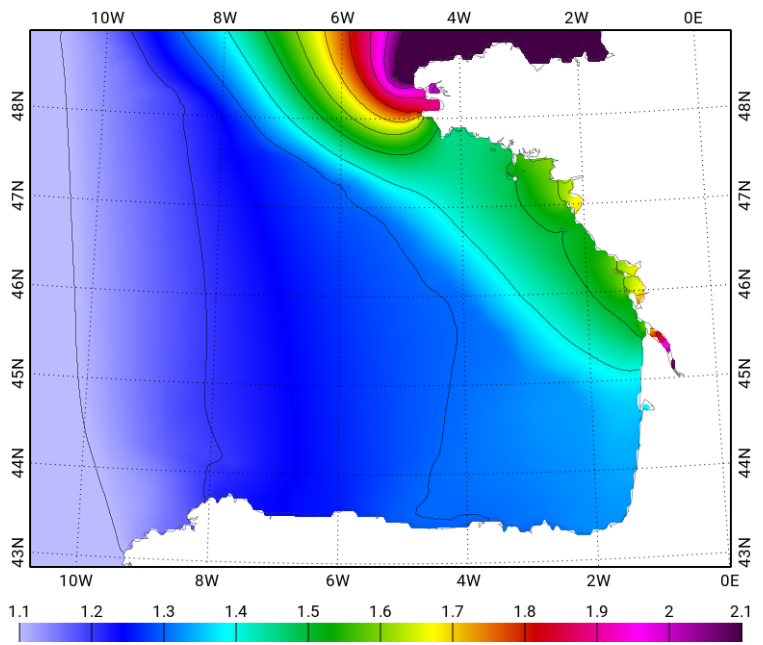

(a)

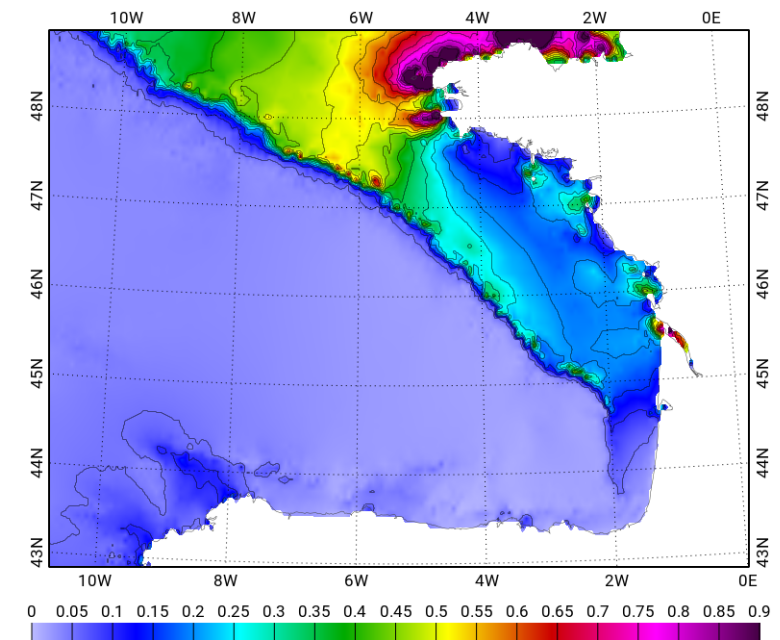

(b)

Figure 1: (a) M2 elevation (m), (b) M2 current (m/s), taken from the FES2012 tidal atlas 
Table 1: Minimum, mean and maximum amplitudes (m) of the tidal elevation for M2, S2, M4 and K1, in the Bay of Biscay, from FES2012

\begin{tabular}{c|c|c|c|c} 
& M2 & S2 & M4 & K1 \\
\hline Mean & 1.36 & 0.48 & 0.035 & 0.070 \\
\hline Min & 1.20 & 0.42 & 0.0070 & 0.063 \\
\hline Max & 2.1 & 0.77 & 0.25 & 0.076
\end{tabular}
our configuration of SYMPHONIE covers the bay from the deep plain to the shelf and coastal shallow waters.

In the first part of this paper, the configuration applied to the Bay of Biscay and the data used to assess the solution are presented. The three strategies chosen to constrain the $3 \mathrm{D}$ circulation model boundaries with tides are then detailed. The 3rd and 4th section are dedicated to the performance evaluation of the different tidal boundary conditions. First, the forcing solutions are compared, followed by the 3D circulation simulations, which are evaluated in two stages: the tidal solution, with respect to tidal elevations and currents; the "global" simulation, comparing more integrating variables (SST, SSS, SSH and total currents). These results are then discussed before concluding.

\section{Model and data}

\subsection{The SYMPHONIE model and the BOBSHELF configuration}

In this section, the SYMPHONIE code used for this study is presented, as well as the BOBSHELF grid and configuration, which is an application of SYMPHONIE to the Bay of Biscay.

\subsubsection{The SYMPHONIE circulation model}

The SYMPHONIE model is based on the Boussinesq hydrostatic equations of momentum, temperature and salinity. The primitive equations are discretized and solved on an Arakawa C-grid, using an energy conserving finite difference method described in Marsaleix et al. (2008, 2009, 2012). Following Damien et al. (2017), horizontal advection and diffusion of momentum are respectively computed with a 4th order centered and a bi-harmonic scheme, while vertical advection of momentum is given by a 2 nd order centered scheme. Advection and diffusion of tracers are computed using the QUICKEST scheme (Neumann et al., 2011). A wet and drying scheme is used with the same vertical coordinates; when the water column thickness drops below $1 \mathrm{~m}$, the wetting and drying algorithm freezes the tracers and cancels out the baroclinic velocities, which means that the model actually becomes locally a 2D barotropic model. The k-epsilon turbulence closure scheme is implemented as in (Michaud et al., 2012).

Large scale forcing terms can be provided to the model. The barotropic tidal forcing consists of the harmonic tidal components provided by an external tidal dataset, introduced through the open boundary conditions, and of 
the astronomical tide potential, implemented in the momentum equations according to Pairaud et al. (2008). The numerical scheme for the open boundary conditions is described in (Marsaleix et al., 2006); their implementation in the present configuration is explained in the Appendix.

The air-sea fluxes are computed with the bulk formulae of Large and Yeager (2004) and variables from an atmospheric model. The daily river discharges are prescribed at the mouth of the rivers or in estuaries and converted into horizontal depth-averaged currents.

\subsubsection{The BOBSHELF grid and configuration}

The Bay of Biscay configuration used in this study is discretized on a curvilinear horizontal grid, implemented using the equations and scaling factors described by Madec (2008) (Figure 2a). The resolution in the region of the 'Pertuis Charentais' and the Gironde estuary is of the order of $300 \mathrm{~m}$. Around Brittany, in the northern part of the domain, the resolution is degraded. Therefore, this area will not be further discussed in this paper. The bathymetry is obtained by merging a GEBCO dataset with several local databases (F. Lyard, pers. comm., 2016). The small scales are removed by smoothing. The bathymetry is however not thresholded, in order to be able to represent the intertidal zones using the wetting drying scheme of the model. This configuration allows the representation of physical processes occurring at different spatial and temporal scales, from the deep plain to shallow areas. In this configuration, large scale processes such as tides and shelf circulation can be studied, as well as fine scale processes like waves or river plume dynamics.

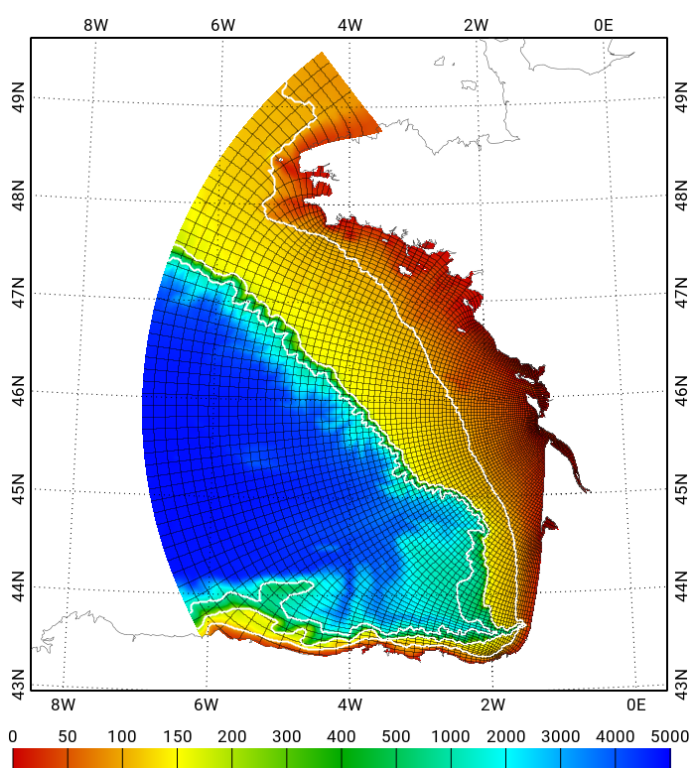

(a)

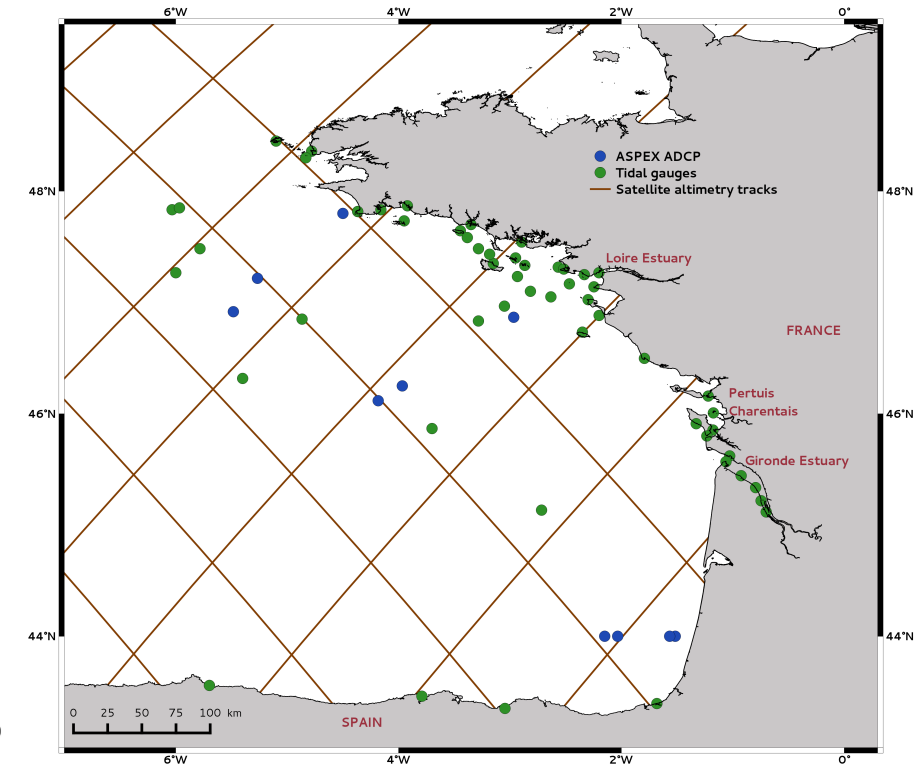

(b)

Figure 2: (a): Bathymetry and grid used for the BOBSHELF configuration. In white : 100, 200 and 1000m isobaths. The displayed grid corresponds to the resolution divided by 5 . (b): Location of the main in situ data of satellite altimetry tracks used for the model assessment 
For the 3D configuration used in SYMPHONIE, generalized sigma coordinates are used on the vertical, with 55 levels. The atmospheric forcing variables are provided by the ECMWF operational analyses (6-h fields, $10 \mathrm{~km}$ resolution). The daily river discharges for 6 rivers are obtained from hydrological stations and retrieved through the French national service 'Banque Hydro' (http://www.hydro.eaufrance.fr/).

For the tidal circulation, tidal elevations and tidal horizontal currents averaged over the water column are prescribed at the open boundaries, for 9 constituents: M2, S2, N2, K2, K1, O1, P1, Q1 and M4.

For the 3D non-tidal circulation, the operational product of MERCATOR-Ocean (the 'IBI' product, with a resolution of $1 / 36^{\circ}$, see for instance Maraldi et al. (2013)) is prescribed at the open boundaries. The resolution near open boundaries is about $3 \mathrm{~km}$, which is matching the IBI resolution. The IBI forcing consists of daily fields of temperature, salinity, horizontal velocities and SSH. These fields are averaged over $25 \mathrm{~h}$ hours to remove the M2 signal. Consequently, most of the tidal signal is filtered out, but we expect some to remain; this question is addressed in section 5 .

The BOBSHELF configuration used for this study was conceived to study fine scale processes occurring in the Bay of Biscay, especially the interactions between the Gironde estuary and circulation on the shelf. Going forward, this modelling setup will be used in particular to assess the observability of such processes by the future satellite altimetry mission SWOT (Fu et al., 2012; Rodriguez et al., 2017) for oceanography and continental hydrology. In this context, the question of tides is central, for two reasons. First, tides play a key role in estuarine dynamics by influencing mixing, resulting in a stronger or weaker stratification at the outlet, and determining the characteristics of the water masses that can interact with the shelf circulation. It is then essential to have the best possible representation of tides to study the estuary to ocean continuum. Secondly, as the SWOT mission is being prepared, increasing efforts are made to provide the best corrections for the different sources of error, including tides. Since one of the objectives of SWOT is to observe small scale processes close to the coast, i.e. where the tides influence on the sea surface height is increased, the quality of satellite altimetry data is dependent on the quality of tidal corrections. A good representation of tidal downscaling up to very coastal areas is then essential to the success of SWOT.

Although the prime focus of this study is tides, we also discuss the performance of the simulations regarding salinity and temperature. Comparisons with buoy SST and SSS are included in Table 2, to show the ability of the model to correctly reproduce salinity and temperature. The mean error compared to satellite SST (L3S product, Orain (2016)) is also estimated at $0.53 \mathrm{C}$ for the years 2011 and 2012. For this calculation, only the dates when the data coverage was higher than $50 \%$ are considered. Finally, the averaged error on SSS when compared to the gridded monthly SSS product in the Atlantic Ocean (Reverdin et al., 2007; Alory et al., 2015) is estimated at 0.19 psu, also for 2011 and 2012.

\subsection{T-UGOm hydrodynamic model}

T-UGOm is a 2D/3D unstructured grid model developed at LEGOS. It can accommodate a variety of numerical discretization (continuous and dis-continuous finite element, finite volumes) on triangle or quadrangle elements. It 
Table 2: Model - data comparison with SST and SSS buoys

\begin{tabular}{c|c|c|c} 
& Oléron & Yeu & Houat \\
\hline RMSE SST $\left({ }^{\circ} \mathrm{C}\right)$ & 0.41 & 0.56 & 0.41 \\
\hline RMSE SSS (psu) & 0.38 & 0.37 & 0.61
\end{tabular}

can be used in time-stepping (TS) or frequency-domain (FD) mode. Both TS and FD are routinely run (Pairaud et al., 2008; Stammer et al., 2014) for operational ocean high frequency signal (i.e. tides and storm surges) corrections in satellite altimetry and gravimetric observations. Initially, the frequency-domain mode has been implemented in the original time-stepping T-UGOm code to dynamically downscale tidal boundary conditions for the time-stepping simulations. For instance, a Flather open boundary conditions setting needs both tidal elevation and currents to be known at the open limits. Where direct interpolation from a global atlas for elevation will be quite suitable, tidal currents (when available) are much less likely to be consistent with the nested grid and bathymetry. The frequencydomain solver, which is based on a wave equation where tidal currents are not necessarily prescribed along open boundaries, allows for reconstructing at a very limited numerical cost a consistent tidal currents field on the nested configuration, open boundaries included.

The T-UGOm FD 2D solver is originally inspired from the CEFMO frequency-domain tidal model that was earlier used for the FES atlases (such as FES2004). FD solver is run for each tidal component separately, it basically assembles a frequency-domain wave equation and the solution is obtained by a simple inversion of the system. Naturally, FD solver is based upon linearized equations, and subsequently non-linear processes require an iterative approach to converge toward the fully non-linear solutions. The number of iterations is rather limited for the major astronomical tidal components; it tends to increase when addressing compound and non-linear tides. In any case, the numerical cost of the FD solver is extremely small compared to the TS solver cost (more than 1000 times smaller). In terms of solution accuracy, the FD and TS solvers are quite equivalent, with of course a limited advantage to the TS solver in non-linear tides cases. Another major advantage of the FD solver reduced numerical cost is the possibility to conduct a wide range of experiments in order to (globally or regionally) calibrate the model parameters such as bottom friction and internal tide drag coefficients, verify bathymetry improvements, or test numerical developments. It must be noticed that the optimal parameters setting for the FD mode will also meet TS mode requirements.

The most commonly used elements in T-UGOm are triangles elements, as they offer the most flexible way to discretize the modelling domain with locally adapted resolution. The obvious purpose of implementing quadrangle elements is to be able to run T-UGOm FD solver on structured grids, enabling T-UGOm tidal solver to be run on most of present structured model configurations. In addition, and in the objective to perform the most consistent tidal downscaling, the elevation and current discretization must fit as close as feasible the usual C-grid discretization. 


\subsection{In situ and satellite data for model assessment}

Several datasets are used to evaluate the performance of the different simulations (Figure 2b). Along-track tidal harmonics obtained from a 21-year long time series of satellite altimetry data from TOPEX/Poseidon, Jason-1 and Jason-2 missions are provided by the CTOH-LEGOS (Birol et al., 2016). The data coverage, extending over the whole domain, from the deep plain to the shelf, makes it a very valuable dataset of 'spatial tidal gauges' for model assessment. We also use tidal constituents computed from shorter time-series stemming from the T/P and Jason-1 tandem missions (10/2002-10/2005 and 02/2009-03/2012) on the 'interleaved' tracks; the latter are located midway along the original tracks thus improving the spatial resolution temporarily. Because of the shorter time-series, the accuracy on those tidal constituents is degraded but still convenient for the purpose of our comparisons. In the Bay of Biscay, the harmonic analysis provides M2 tidal elevations with uncertainties of $0.26 \mathrm{~cm}$ and $0.39 \mathrm{~cm}$ for the nominal and tandem mission respectively; such values are very low compared to the M2 elevation that ranges between 1.20 $\mathrm{m}$ and $2.10 \mathrm{~m}$ in our domain (with a mean value of $1.36 \mathrm{~m}$ ). These estimations represent the error on the harmonic analysis in itself. The error is linked to the method that is used to extract the aliased frequencies as precisely as possible from the ocean background signal. Altimetric data close to the coast undergo a strong loss of accuracy for several reasons due to instrumental errors and inaccuracies on geophysical corrections; therefore no data is available at a distance of roughly $50 \mathrm{~km}$ from the coast.

Tidal gauges from the REFMAR, SPC Gironde and Puertos del Estado networks provide both tidal harmonics and, for a few of them, SSH time series. Other tidal gauges, previously used in Pairaud et al. (2008), also provide tidal harmonics. These data were obtained through the French Navy and the OHI, but they do not include any indication of error on the tidal analysis.

Harmonic analyses are performed on ADCP current data provided by IFREMER and obtained during the ASPEX campaign (Le Boyer et al., 2013; Kersalé et al., 2016), for comparison with tidal current harmonics calculated at the same positions in the model. 2D mean daily currents, projected on the along-shore and cross-shore axes are also calculated and compared.

Because tides impact the regional hydrology through mixing and current rectification, we also compare model outputs with temperature and salinity data. We use the CORA-IBI database (temperature and salinity profiles) by IFREMER (Szekely et al., 2017), the Islands network (IFREMER), Météo-France buoys, and the Puertos del Estado network (sea surface temperature and salinity). A gridded $\left(1^{\circ} \times 1^{\circ}\right)$ database giving monthly estimates of the SSS (sea surface salinity) in the Atlantic Ocean (Reverdin et al., 2007; Alory et al., 2015) is also used.

\section{Tidal open-boundary conditions}

\subsection{The different strategies for tidal OBC tested in this paper}

The OBC in SYMPHONIE are based on Flather and radiation conditions whose implementation is described in the Appendix. External information is needed to specify incoming information. For the barotropic tides, the external 
information consists in the elevations and horizontal currents averaged over the water column for the nine main tidal constituents in the Bay of Biscay: M2, S2, N2, K2, O1, P1, K1, Q1, M4. For the non-tidal circulation, it consists in sea surface elevation, 3D temperature and salinity fields, 3D horizontal currents fields for the residual circulation, provided by MERCATOR-Ocean (as described in 2.1.2).

In this paper, we compare three 7-month simulations of SYMPHONIE 3D, each one forced by a different tidal solution (elevation and horizontal depth-averaged currents): 1/ FES2012 atlas, 2/ solution from a SYMPHONIE 2D simulation, 3/ solution from a T-UGOm spectral simulation. The performed simulations are summarized in Table 3. We emphasize the fact that the only differences between the three runs (S3D_FES, S3D_Tugo and S3D_S2D) are on the source of the tidal elevations and currents prescribed at the open boundaries. The OBC numerical scheme is the same for the three 3D SYMPHONIE runs.

Table 3: Summary of the performed simulations

\section{D - forcing solutions}

\begin{tabular}{|c|c|c|c|}
\hline $\begin{array}{l}\text { Tidal forcing } \\
\text { (elevations) }\end{array}$ & Model used & Simulation name & Characteristics \\
\hline FES2012 & SYMPHONIE & S2D & $\begin{array}{l}\text { 2D clamped } \\
\text { No OGCM or atmospheric forcing }\end{array}$ \\
\hline FES2012 & T-UGOm & Tugo & $\begin{array}{l}2 \mathrm{D} \\
\text { Spectral }\end{array}$ \\
\hline \multicolumn{4}{|l|}{ 3D - circulation solutions } \\
\hline $\begin{array}{l}\text { Tidal forcing } \\
\text { (elevations and currents) }\end{array}$ & Model used & Simulation name & Characteristics \\
\hline FES2012 & SYMPHONIE & S3D_FES & \multirow{3}{*}{$\begin{array}{l}\text { 3D } \\
\text { OGCM and atmospheric forcing }\end{array}$} \\
\hline S2D & SYMPHONIE & S3D_S2D & \\
\hline Tugo & SYMPHONIE & S3D_Tugo & \\
\hline
\end{tabular}

\subsection{FES2012 atlas}

FES2012 is a recent version of the FES (Finite Element Solution) global tidal model (Carrère et al., 2012), following the FES2004 version (Lyard et al., 2006). This model is based on the T-UGOm model (frequency-domain solver for the astronomical tides, and time-stepping solver for the non-linear tides) and assimilates tide gauges and satellite altimetry derived harmonic constants. Errors both in prior and assimilated solutions have been significantly reduced compared to FES2004, especially on the coastal and shelf areas, thanks to a longer time series of altimetric data, a more precise bathymetry, and the use of improved data assimilation schemes. The latest FES atlas (i.e. FES2014), 
despite of superior accuracy, was not used to keep consistent with some already existing simulations forced with FES2012.

\section{3. $2 D$ simulations}

As an alternative to the FES2012 tidal atlas, two other tidal forcings are generated to force the 3D circulation model. The main advantage of these forcings is that they are generated on the same grid (BOBSHELF) and with the same bathymetry as the ones used in the 3D simulations.

To generate tidal boundary conditions, both for tidal elevations and currents, it is possible to run simplified (no atmospheric or OGCM forcing) 2D simulations with SYMPHONIE. These simulations are performed with clamped (or Dirichlet) conditions, meaning that only tidal elevations are used at the boundaries (in our case, FES2012 tidal elevations). Tidal currents are not considered. The model is run for 7 months, from October 2010 to April 2011, with 9 tidal constituents (M2, S2, N2, K2, K1, O1, P1, Q1 and M4). The harmonic analysis is run online. 7 months is the necessary period to be able to separate the different waves.

For the 2D T-UGOm spectral simulations (called Tugo hereafter), the clamped conditions and FES2012 tidal elevations are also used at the open boundaries. For consistency, the bottom friction is set at the same value in T-UGOm as in SYMPHONIE.

In the next section, we compare and evaluate the three tidal boundary conditions (FES2012, S2D and Tugo) by comparing tidal elevations to the available observations. In section 4 , the results of the 3D simulations are detailed, first focusing on the tidal solution (elevation and currents), then on a wider range of parameters, to assess the influence of the tidal forcing on the global circulation.

\subsection{Assessment of the tidal forcing solutions}

Comparisons with tidal harmonics from satellite altimetry data and tidal gauges are presented in Figure 3. These bar plots represent the mean complex error, which accounts for errors both in amplitude and in phase. The complex error $H_{s}$ is calculated as follows:

$$
\begin{aligned}
H_{s} & =\sqrt{h_{1}^{2}+h_{2}^{2}} \\
h_{1} & =H_{m} \cos \left(G_{m}\right)-H_{o} \cos \left(G_{o}\right) \\
h_{2} & =H_{m} \sin \left(G_{m}\right)-H_{o} \sin \left(G_{o}\right)
\end{aligned}
$$

With $H_{m}$ and $G_{m}$ the amplitude and phase of the modelled constituent, and $H_{o}$ and $G_{o}$ the amplitude and phase of the observed constituent.

Satellite altimetry results (Figure 3b) show a better agreement with FES2012 for M2 and M4. This is expected because of the assimilation of these data in FES2012. The S2 tide is better represented by S2D and Tugo. This is because the S2 signal captured by the altimetry can be divided into two parts: an astronomic one, and an atmospheric 
one, which is partly non-stationary. As a consequence, the signal assimilated in FES2012 corresponds to a sort of S2 'average' that does not take into account the seasonal variability of this constituent. This can explain why the performance of the S2 assimilation is relatively limited compared to that of other constituents. More details can be found in Lyard et al. (2006).

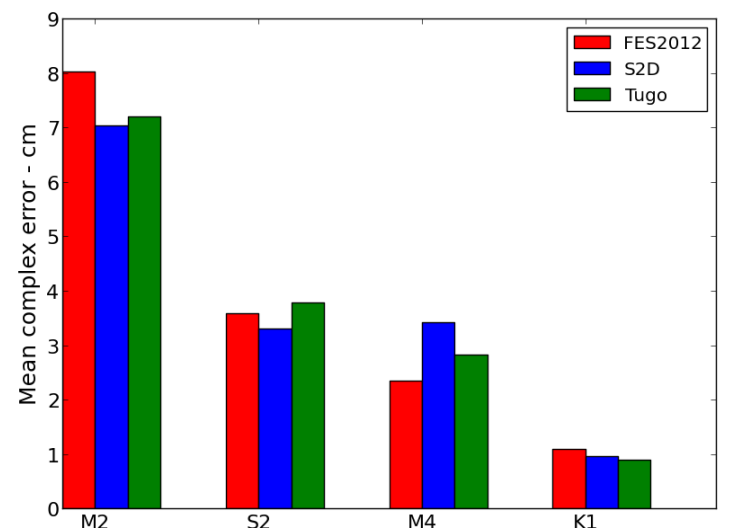

(a) Shelf and coastal tidal gauges

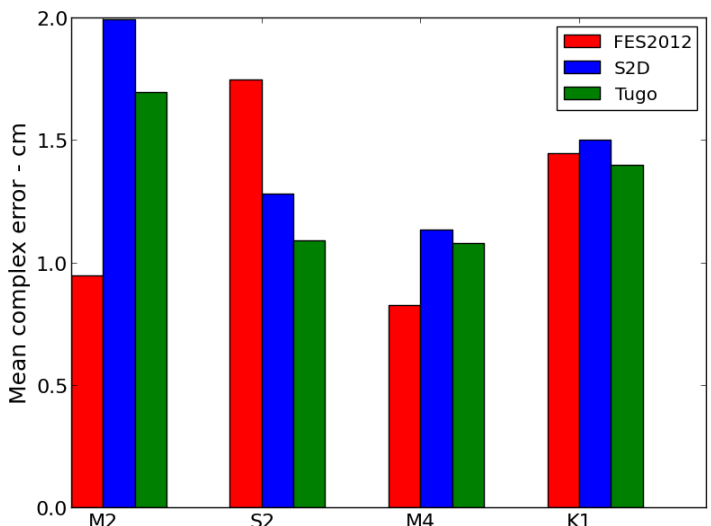

(b) Satellite altimetry: nominal and interlaced tracks

Figure 3: Mean complex errors (in $\mathrm{cm}$ ) over the BOBSHELF domain for the M2, S2, M4 and K1 harmonics, between the forcing solutions FES2012, S2D and Tugo, and available observations

Tidal gauges results (Figure 3a) suggest that the regional models are more accurate near the coast for the M2 tide. However, the M4 tide is better represented by FES2012. M4 is generated by the interaction of M2 with itself. Therefore, a part of the errors on M4 is a direct consequence of the errors on M2. In FES2012, the assimilation is performed independently for each constituent, meaning that the M2 solution is not directly impacting the M4 solution, reducing the level of errors on this constituent. In S2D and Tugo, there is no assimilation, which means that the M4 errors are partly inherited from M2, and squared.

For K1, the level of error is similar for the three solutions and for the two datasets (tidal gauges and satellite altimetry). In the Bay of Biscay, the signal to noise ratio in satellite altimetry is smaller for K1 than for M2, because the K1 tide has a much weaker amplitude and a much lower aliased frequency (180 days against 62). As a consequence, assimilating the $\mathrm{K} 1$ altimetric signal has almost no impact in this region.

As shown in Figure 3, the level of error between the two data-model comparisons (tidal gauges and satellite altimetry) is significantly different. For example, the M2 complex error is multiplied by more than 4 (for Tugo). Tidal gauges are mostly located on the coast, and a few are on the shelf. In these areas, the tidal signal is also amplified when compared to the open ocean, where most of the satellite data are obtained from. For instance, the M2 amplitude is doubled between the open ocean and certain coastal areas. In addition, near the coast, the evolution of the tidal signal is very sensitive to the geometry of the area (coastline, bathymetry). This means that the signal can be significantly different between two close locations, whereas in the open ocean, tides are relatively homogeneous in space. This 
strong spatial variability is difficult to reproduce in a model where the mesh size is larger than the length scale of bathymetric features. One model cell is then susceptible to discretize an area where the tidal signal would present gradients at a smaller scale.

\section{Assessment of the 3D SYMPHONIE simulations}

In this section, we evaluate the impact of using different tidal boundary forcing on the 3D SYMPHONIE model results by comparing the simulations to observations from different data sets. The simulation closest to the observations (within the data uncertainties range) is obviously identified as the most realistic one. These comparisons also stand for a more general assessment of the BOBSHELF configuration: we show indeed that the model-data misfits for the different variables are low over the period of study which makes us confident in the ability of the BOBSHELF configuration to simulate the main processes of the Bay of Biscay circulation with the needed accuracy for our purposes (section 2.1.2).

\subsection{Tidal elevations}

Complex errors between modelled and observed tidal amplitudes and phases are given in Table 4. Misfits from M2 altimetry are reduced by more than $75 \%$ between S3D_FES and S3D_Tugo (70\% for S3D_S2D). For the tidal gauges, the error is reduced by $20 \%$ (13\% for S3D_S2D). Altimetry errors are also lower for S2 with the Tugo and S2D forcings. For M4 and K1, the errors on altimetry are slightly higher for S3D_S2D and S3D_Tugo than for S3D_FES (between 8 and 15\%). However, the difference is more significant for the tidal gauges comparison on M4 (more than +38\%). For K1, S3D_S2D and S3D_Tugo simulations perform a little bit better than S3D_FES (between -11\% and $-17 \%)$.

Table 4: Mean complex errors $(\mathrm{cm})$ between the 3D circulation solutions and the available data for M2, S2, M4 and K1. SA = Satellite altimetry ; TG = Tidal gauges

\begin{tabular}{l|cc|cc|cc|cc|}
\multirow{2}{*}{ 3D simulations } & \multicolumn{2}{|c|}{ M2 } & \multicolumn{2}{|c|}{ S2 } & \multicolumn{2}{c|}{ M4 } & \multicolumn{2}{c|}{ K1 } \\
& SA & TG & SA & TG & SA & TG & SA & TG \\
\hline S3D_FES & 7.49 & 9.30 & 1.89 & 3.42 & 0.993 & 2.94 & 1.29 & 1.28 \\
\hline S3D_S2D & 2.10 & 8.03 & 1.56 & 3.80 & 1.15 & 4.40 & 1.49 & 1.06 \\
\hline S3D_Tugo & 1.84 & 7.39 & 1.55 & 3.73 & 1.11 & 4.07 & 1.42 & 1.13
\end{tabular}

A more detailed view of the complex errors on M2 is available in Figure 4: all the tidal gauges (shown in Figure $2 b)$ used to compute the mean error are represented, from the northern to the southwestern limit of the domain. All tidal gauges between Ouessant and Gijon are either directly on, or very close to the coast (depth $<50 \mathrm{~m}$ ). The T1 to 'COURIR5 3' gauges are located on the shelf (data from Le Cann (1990) and SHOM dataset). 'MGFCOR large' is the only gauge in the deep plain (depth $>4000 \mathrm{~m})$. 
This figure is consistent with the results presented in Table 4: M2 errors are globally much lower in S3D_Tugo and S3D_S2D than in S3D_FES. Out of 52 tidal gauges, the M2 complex error is the highest for S3D_FES in 33 of them. These errors also show a strong spatial variability. Tidal gauges within a few kilometers of each other can display a very different trend. For example, the 'Birvideaux' and 'Vilaine P1' tidal gauges are distant of less than $10 \mathrm{~km}$ (see Figure 5 for locations). The first one shows that S3D_FES has the highest error, whereas the second one suggests the opposite. This underlines the difficulty to compare single-point data like tidal gauges records to numerical simulations, as already discussed in the previous section.

In this sense, satellite altimetry data appear to be more useful. First, it provides a spatially homogeneous dataset that covers a larger area, allowing us to check if the data is consistent within a few kilometers. In terms of data quality, it is also important to notice that tidal gauges data are obtained from different providers, with differences in instrumentation and data processing. It is of course necessary to use tidal gauges to evaluate regional models close to the coast. In this modelling configuration in particular, the resolution is increased near the coast. Tidal gauges comparisons must be made, because satellite altimetry cannot yet provide reliable data in these areas. However, it is important to be aware of the challenges associated with these data, that can seem easier to use than satellite altimetry at first.

The Royan and Port-Bloc tidal gauges are located at the mouth of the Gironde estuary, each on one side of the river. The Richard, Lamena, Trompeloup and Fort-Médoc gauges are distributed within the estuary, Fort-Médoc being the most upstream point. At the Royan, Port-Bloc and Lamena stations, S3D_FES seems to provide the best solution, but this tendency is reversed when moving upstream, from Lamena to Fort-Médoc. The Gironde estuary is a particular environment, with the presence of a turbidity maximum and fluid mud (Sottolichio and Castaing, 1999). The latter can induce large variations in bed roughness, which influences tidal propagation and distortion within the estuary: in the presence of fluid mud, the bed roughness is very low, inducing an increase of velocities, and a reduced damping of the M2 tide. In order to correctly represent tides within the estuary, bottom friction tuning will be necessary. In our configuration where no specific tuning is done (that would be out of the scope of this paper) and in which sedimentary processes are not taken into account, we cannot expect to represent accurately tides in the estuary. The fact that S3D_FES is closer to the observations in the lower estuary than S3D_Tugo and that the opposite is found in the upper estuary seems paradoxical. However, given the limitations of the configuration there, we suggest that error compensating effects are responsible for the good match of S3D_FES with observations at the estuary mouth. 


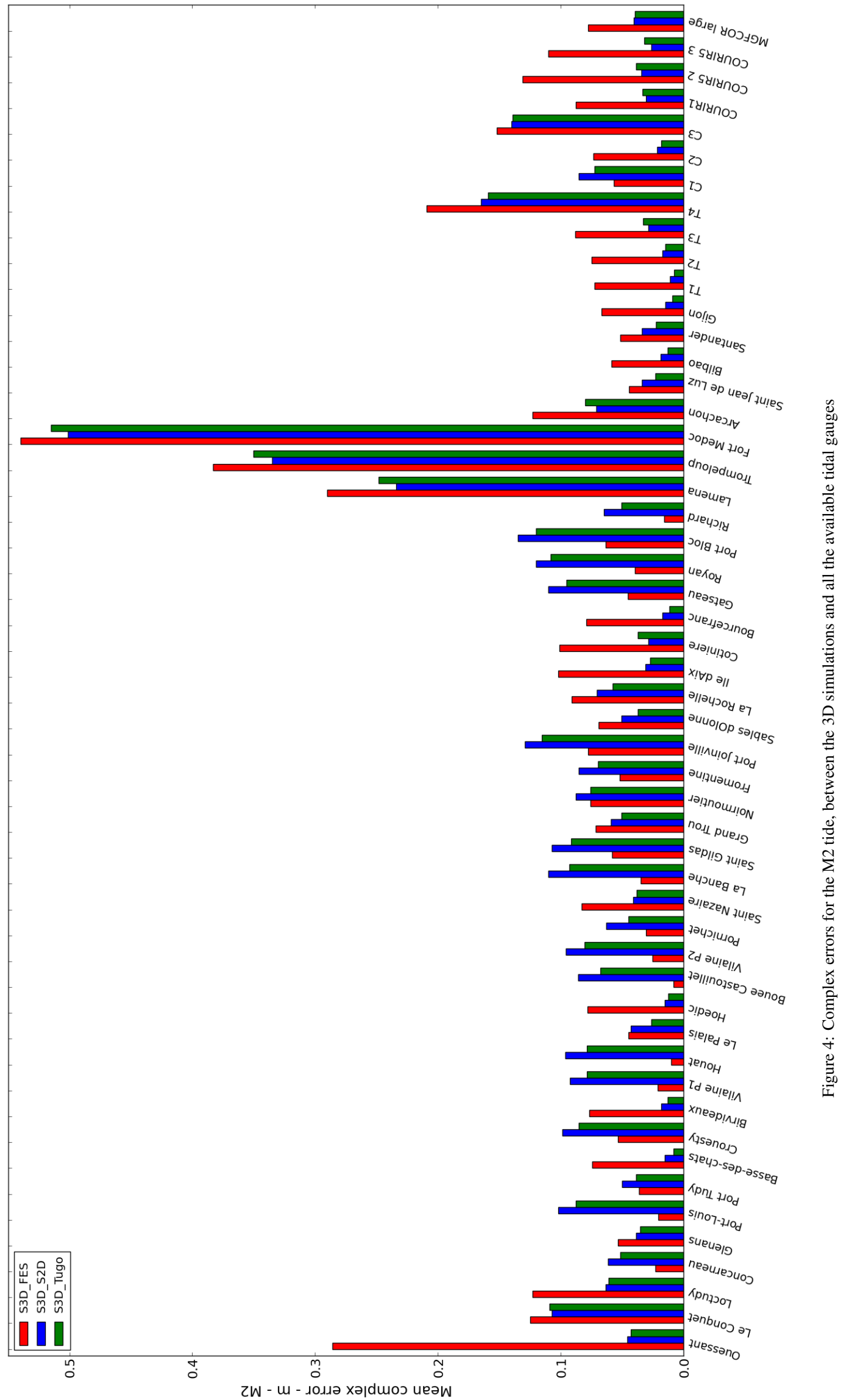


Figure 5 represents the differences in amplitude and in phase, for M2, between S3D_Tugo and S3D_FES. In amplitude, the highest differences (more than $7 \mathrm{~cm}$ ) are obtained on the shelf, in the northern part of the domain, where the tides are highly energetic. Overall, the difference is close to $5 \mathrm{~cm}$ in amplitude. This value is decreasing within the Gironde estuary, suggesting again that the dynamics inside the estuary are more constrained by local effects (bathymetry, friction), than by the remote forcing. The same behavior is obtained for the M2 phase difference, which is the lowest inside the estuary.
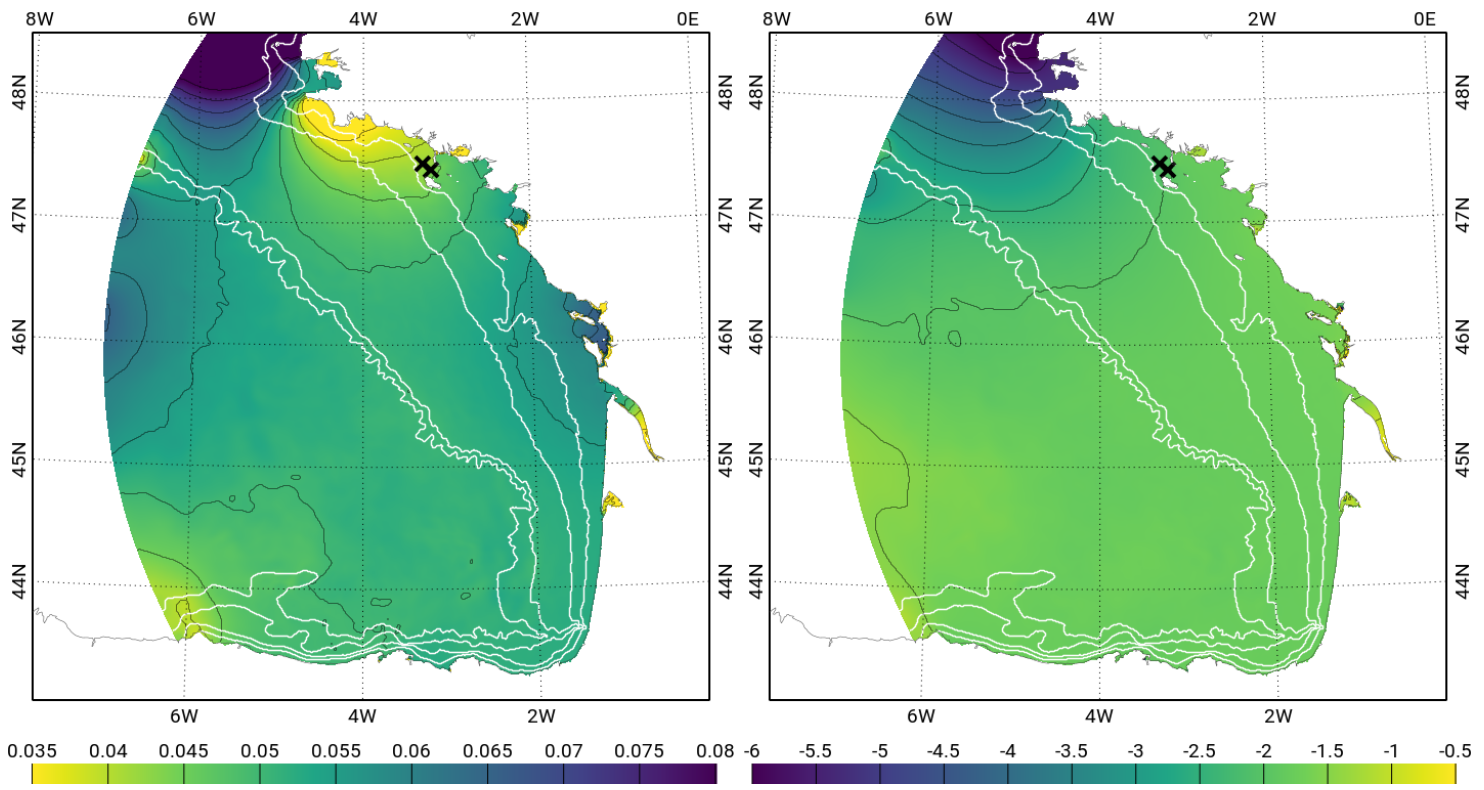

Figure 5: Difference between S3D_Tugo and S3D_FES for the M2 amplitude (left, $\mathrm{m}$ ) and phase (right, ${ }^{\circ}$ ). In white: 50, 100, 200 and 1000m isobaths. Black crosses: 'Vilaine P1' and 'Birvideaux' tidal gauges.

\subsection{Tidal currents}

Tidal analyses are performed on ADCP data obtained during the ASPEX campaign (Le Boyer et al., 2013; Kersalé et al., 2016), and tidal ellipses parameters are then calculated. 3D velocity currents are averaged over depth to perform a 2D analysis. No evidence of a significant vertical structure of the tidal currents was found. For consistency, only the ASPEX ADCP data covering the 7 months simulation period are used for comparison.

Figure 6 shows a global good agreement between the three S3D simulations and ASPEX data, both for M2 and M4, making it difficult to draw conclusions on the best simulation. S2 ellipses (not shown) exhibit results close to the M2 ellipses. Significant direction differences between model and data for ASPEX1 and ASPEX3 are found for M2 (Figure 6a). The M4 tidal ellipses seem a little bit more different between the three solutions (Figure 6b). For ASPEX1, the S3D_S2D and S3D_Tugo ellipses are closer to data than S3D_FES. For ASPEX3, the direction of the ellipse is better reproduced in S3D_Tugo. For ASPEX5 and ASPEX9, the semi-major axis is also closer to data in S3D_Tugo than in the other two simulations. 


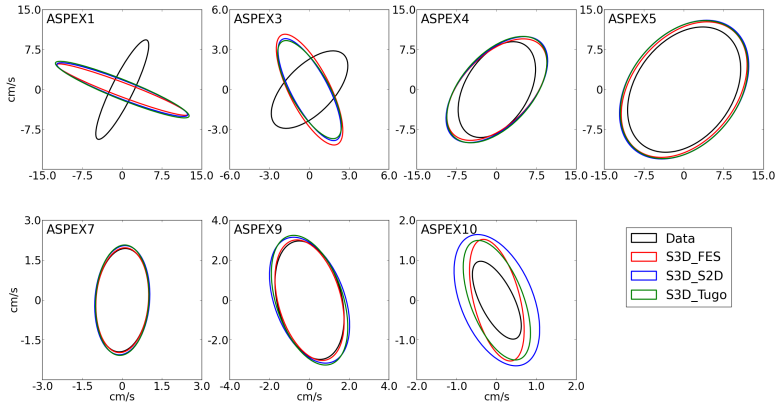

(a) M2
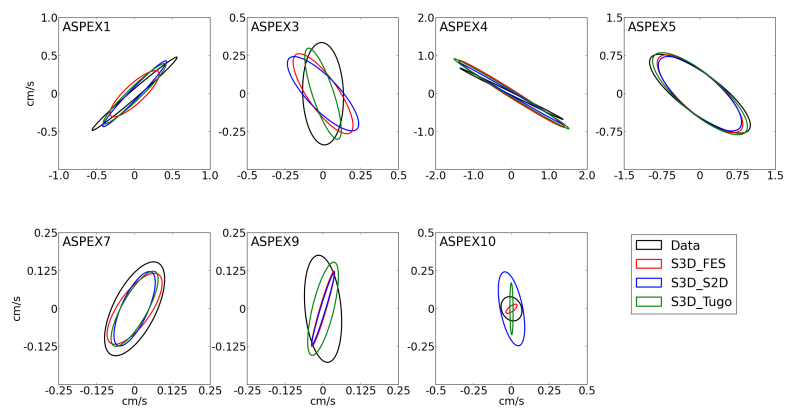

(b) M4

Figure 6: Tidal current ellipses for the M2 and M4 tide: comparison between data and S3D simulations at each ASPEX mooring.

\subsection{Sea surface height}

The SSH time series of 11 tidal gauges are compared to each 3D simulation. Not all the gauges used for the tidal harmonics comparisons provide SSH time series, explaining why the number of data points used here is reduced. Standard deviations (STD) and root mean square errors (RMSE) are normalized (divided by the data standard deviations), to represent all the tidal gauges used on the same Taylor diagram (Figure 7). The three simulations give good results, with correlation coefficients greater than 0.99 and normalized RMSE between 5 and 15. Normalized STD show a greater agreement between data STD and S3D_S2D or S3D_Tugo than S3D_FES.
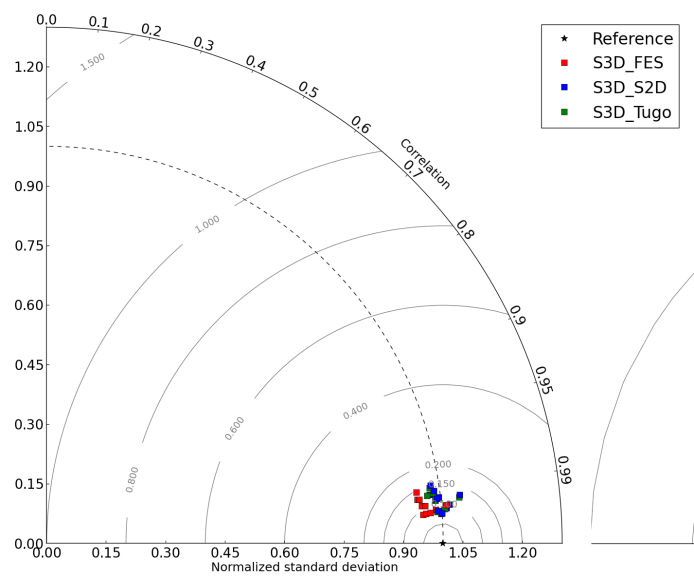

S3D_Tugo

Figure 7: Taylor diagram obtained from the comparison of 11 tidal gauges time series with model outputs. On the right: zoom on the lower right corner.

\subsection{Current velocities}

The ADCP data used previously to compare tidal ellipses are now processed to compare total currents. 2D daily means are calculated in the along-shore and cross-shore directions and compared. Mean RMS errors and correlations are calculated over 9 ADCPs (Table 5). 
Table 5: Model - data comparison between 2D daily means of cross-shore and along-shore current velocities

\begin{tabular}{c|cc|cc|cc} 
& \multicolumn{2}{|c|}{ S3D_FES } & \multicolumn{2}{c|}{ S3D_S2D } & \multicolumn{2}{c}{ S3D_Tugo } \\
& $R \overline{M S E}$ & $c \overline{o r r}$ & $R \overline{M S} E$ & $c \overline{o r r}$ & $R \overline{M S E}$ & $c \overline{o r r}$ \\
\hline Along-shore $\left(\mathrm{cm} . \mathrm{s}^{-1}\right)$ & 4.96 & 0.448 & 4.77 & 0.510 & 4.55 & 0.585 \\
\hline Cross-shore $\left(\mathrm{cm} . \mathrm{s}^{-1}\right)$ & 2.94 & 0.125 & 2.89 & 0.188 & 2.95 & 0.202
\end{tabular}

For the along-shore currents, the mean RMSE is lower for S3D_Tugo and S3D_S2D than for the S3D_FES simulation. The difference is rather small (less than $10 \%$ ). However, the mean correlation is increased by more than 30 $\%$ between S3D_FES and S3D_Tugo (14\% for S3D_S2D). The correlations are significant (95 \% confidence level) in 8 out of 9 moorings for S3D_Tugo, against 7 for S3D_S2D and 6 for S3D_FES. Besides, the global numbers of table 5 do not represent local behaviors. The impact of the tidal forcing is indeed different on the different moorings and changing in time. This is illustrated on Figure 8 for the ASPEX9 mooring which is located over the slope at the $44^{\circ} \mathrm{N}$ section: the variability of the signal is better reproduced by the S3D_Tugo simulation, particularly at the beginning and the end of January 2011, and at the beginning of March 2011. To make sure that these differences are not due to very local features, the results are plotted representing the median value of 9 grid points (data co-located grid point and 8 surrounding grid points). The minimum and maximum values from these 9 points are also represented by the shaded area. This type of data-model comparison can be called 'fuzzy verification', and comes from the fact that high resolution models often score poorly with single-point comparisons, while their performance is often very satisfying. Thus, fuzzy verification allows the model to be slightly displaced (here in space), and still be valuable (Ebert, 2008).

Cross-shore currents are very weak $(0-1 \mathrm{~cm} / \mathrm{s}$ on average). Correlation values are not significant and cannot be compared between the different simulations.

\subsection{Temperature and salinity}

Modelled temperature and salinity are evaluated with respect to several databases of surface measurements (Islands network, Puertos del Estado network, Météo-France buoy) and in situ profiles from the CORA-IBI database (Szekely et al., 2017). Time series of model-data comparisons and statistics (RMSE, STD, correlation, not shown) are inconclusive, because the differences between the S3D simulations are within the in-situ measurements uncertainties.

Comparisons with the gridded monthly sea surface salinity product in the Atlantic Ocean (Reverdin et al., 2007; Alory et al., 2015) show some differences between the 3D simulations. For the 7 months runs, considering a 3 months spin-up for the 3D circulation, comparisons are made from January to April. Monthly means of the model SSS are computed over the same grid as the observations. Noticeable differences between the simulations are found for two points only, located on the shelf, and under the influence of the Gironde estuary (Figure 9a, hereafter 2W46N) and Loire estuary (Figure 9b, hereafter 3W47N) plumes.

For the 2W46N location, in January and February, the S3D_FES simulation seems to be in better agreement with 

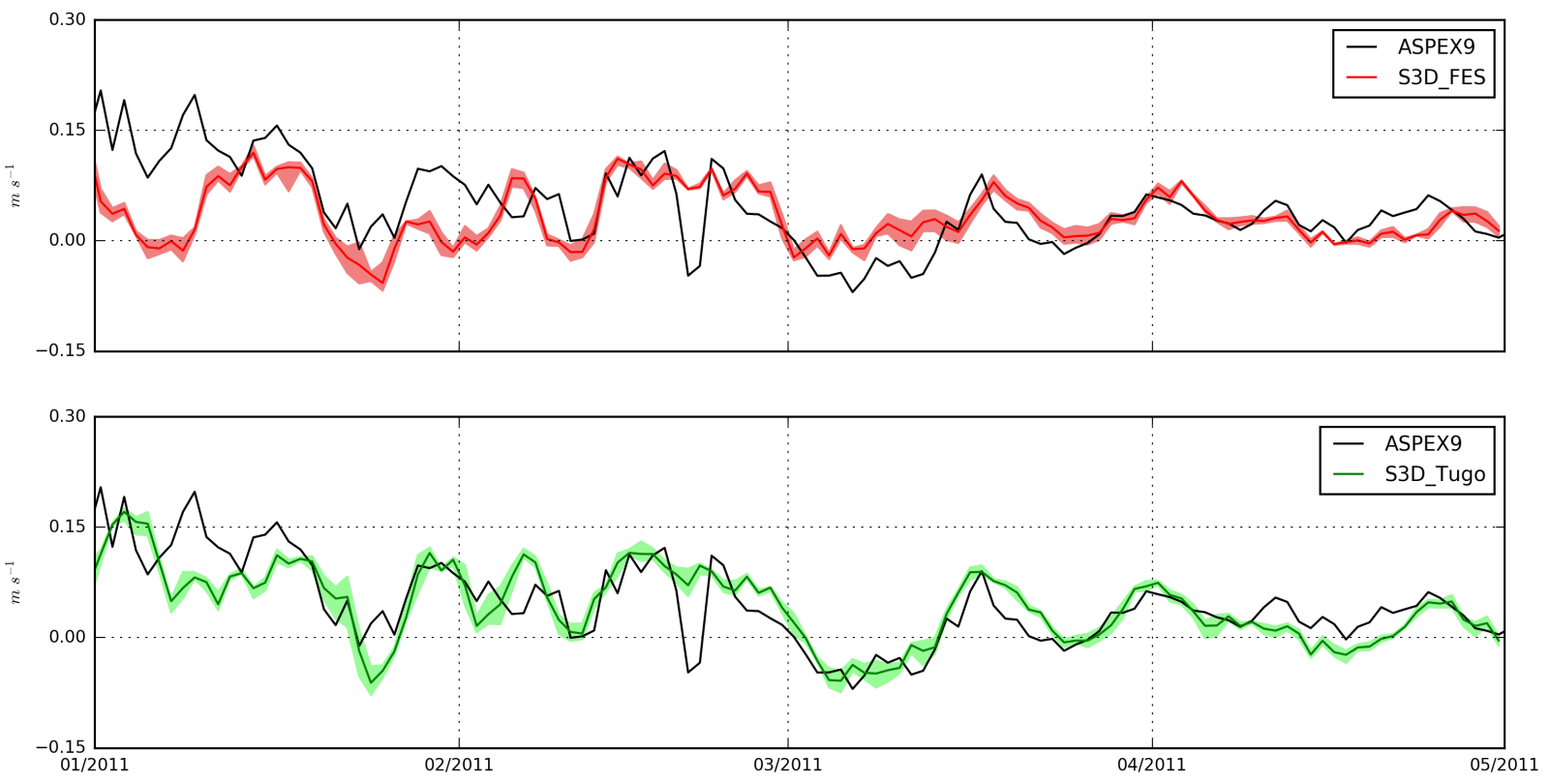

Figure 8: 2D mean daily current velocities for the S3D_FES (top) and S3D_Tugo simulations (bottom), compared to ADCP data from Le Boyer et al. (2013). Solid line: median of 9 grid points. Shaded area: minimum and maximum values from 9 grid points

the observations. On the other hand, in March, the S3D_S2D and S3D_Tugo results are very close to observations. In April, all three simulations show a similar level of performance, although the S3D_S2D and S3D_Tugo simulations are slightly better. For the $3 \mathrm{~W} 47 \mathrm{~N}$ point, S3D_Tugo has the overall lowest error budget, while there is a systematic underestimation of the sea surface salinity for the 3 simulations.

In summary, comparisons of data-model misfits between the 3D simulations do not show strong differences. However, it does not mean that there are indeed no differences in temperature and salinity between these simulations. A more extensive dataset would be necessary to reach a more straightforward conclusion.

\section{Discussion}

\subsection{A downscaling challenge: an accurate representation at all scales}

Based only on the results obtained on the tidal forcings (FES2012, S2D and Tugo), the FES2012 forcing seemed to be the best option, because of a clear lower error budget for M2 when compared to satellite altimetry data. However, the results obtained with the 3D simulations show that the Tugo forcing provides an overall better solution, both for tidal elevations and currents. The difference of performance between the forcing FES2012 and the 3D solution forced by FES2012 (S3D_FES) is particularly striking with respect to satellite altimetry: the complex error is almost multiplied by 8 (compare Figure $3 b$ and Table 4). This gap in performance between FES2012 and S3D_FES can be imputed to the differences in resolution and bathymetry at the open boundaries. In other words, there is an inconsistency between the FES2012 currents at the open boundary conditions and the tidal dynamics inside the domain that 


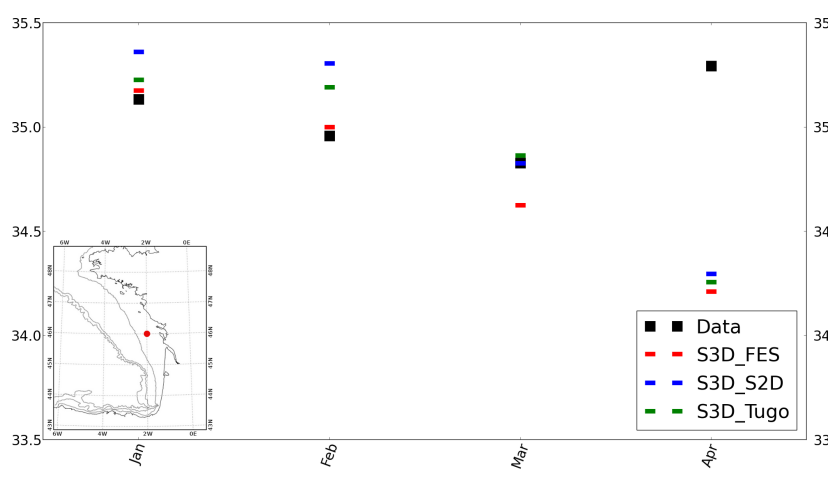

(a) $2^{\circ} \mathrm{W}, 46^{\circ} \mathrm{N}$

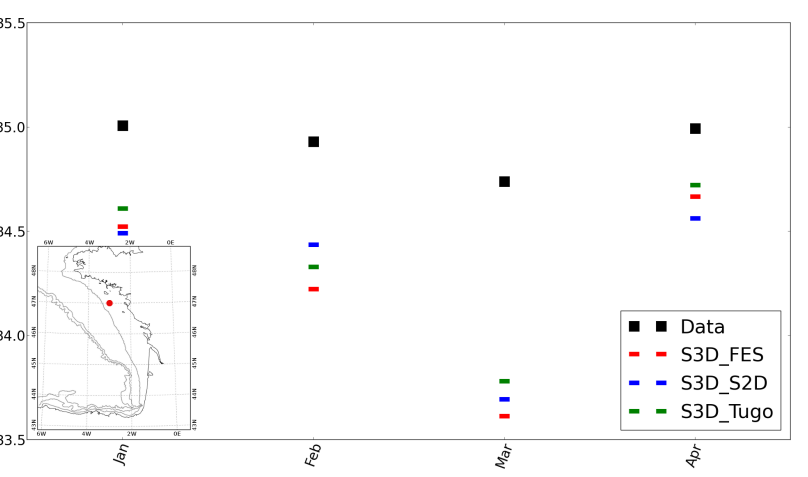

(b) $3^{\circ} \mathrm{W}, 47^{\circ} \mathrm{N}$

Figure 9: Monthly sea surface salinity (psu) comparisons between the Atlantic Ocean database and the S3D simulations, at the two points shown on the maps

are constrained by the resolution and bathymetry of the BOBSHELF configuration. These over-specification errors are mainly impacting the $\mathrm{M} 2$ tides because the $\mathrm{M} 2$ currents are largely dominant.

Over-specification is a recurrent pitfall with OBC. It occurs here because of the high sensitivity of tidal currents to bathymetry and to the detailed representation of the coastline inside the domain. To our knowledge, there is no OBC scheme that prevails to systematically reduce the over-specification errors. Continuous efforts are developed in the community as configurations evolve with higher and higher resolution representing more and more complex processes. The recent study of Herzfeld and Andrewartha (2012) for instance proposes a method based on the Dirichlet conditions in conjunction with a local flux adjustment for volume conservation that has been successfully tested in different configurations and that requires little tuning. Robustness and simplicity are of course highly attractive qualities for a scheme to be implemented in complex systems. Over-specification may be enhanced in the presence of tides together with a low-frequency circulation (such as in our case) because active boundary conditions for tides can be reflective for the low-frequency circulation as noted by Herzfeld and Gillibrand (2015). These authors investigate a method based on dual-relaxation time-scales to solve possible conflicts on the passive/active nature of open boundaries for the different components of the flow. Such new research offers interesting perspectives of improvement for the OBC schemes that we may want to explore in future studies. In the present one, we have chosen to adjust the external forcing field in order to reduce the inconsistencies with the interior solution rather than modifying the $\mathrm{OBC}$ scheme.

The modelled M2 field for S3D_FES and S3D_Tugo is shown in Figure 10: the complex error between these simulation results and the tidal harmonics extracted from satellite altimetry data is represented by the circles superimposed on the maps. Figure 10 shows that the errors are well distributed over the whole domain, and not only at the open boundaries. In other words, the change of tidal open-boundary forcing has a significant impact on SSH all over the basin. More precisely, the M2 amplitude is globally underestimated in S3D_FES, and the M2 phase is overestimated.

The better performance with S3D_Tugo results from the use of forcing tidal currents that are consistent with the interior resolution and bathymetry, since the Tugo model has been run on the BOBSHELF grid. This is illustrated 

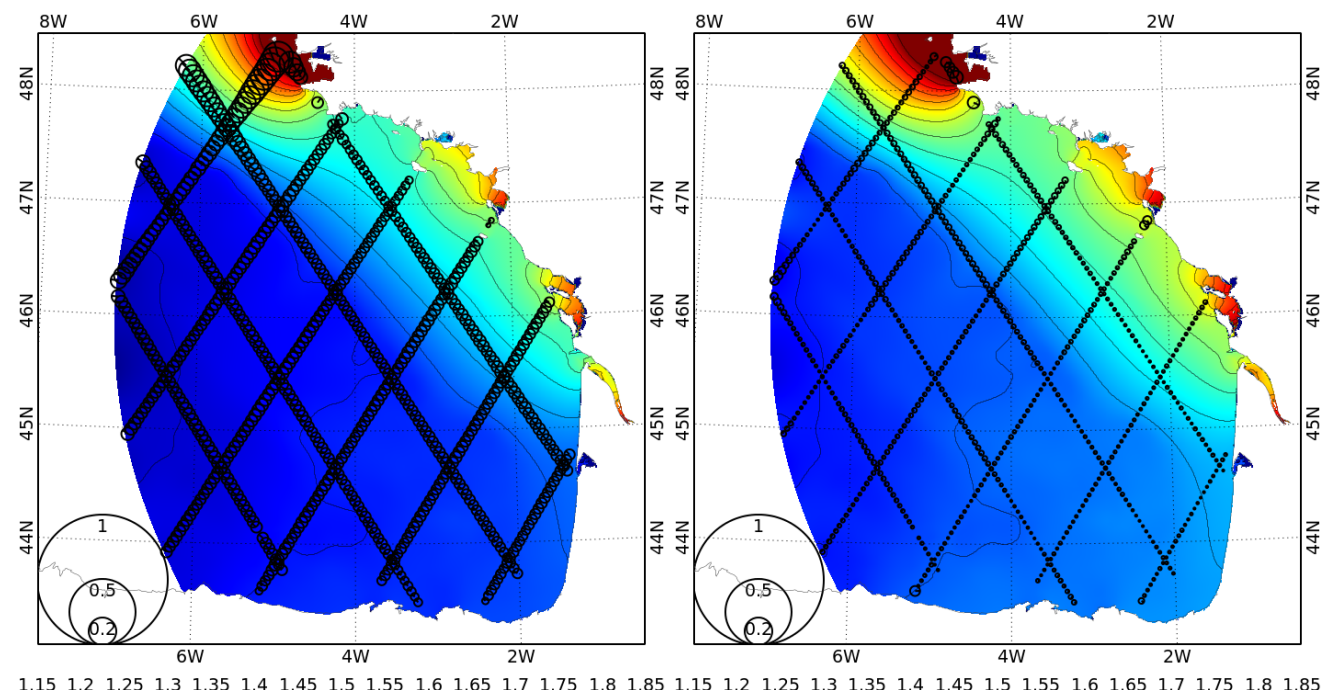

(a) S3D_FES

(b) S3D_Tugo

Figure 10: Comparison between the M2 tide obtained from satellite altimetry and from S3D. In the background: M2 amplitude (m). The circle size is proportional to the complex error $(\mathrm{m})$.

on Figure 11a, showing the difference between the M2 tidal current amplitude in FES2012 and Tugo. Differences larger than $10 \mathrm{~cm} / \mathrm{s}$ are observed over the northern slope and shelf (north of $45^{\circ} \mathrm{N}$ ) where the tidal currents are strong and the resolution increased. In particular, the difference between the 2D M2 tidal current in FES2012 and Tugo can reach more than $20 \mathrm{~cm} / \mathrm{s}$ close to the Armorican slope, at the north-west open boundary of our domain (Figure 11a). Between S3D_FES and S3D_Tugo (Figure 11b), these differences are reduced, but they can still reach more than 10 $\mathrm{cm} / \mathrm{s}$. In the deep plain, the differences are much smaller in the 3D simulations. S3D_S2D also gives better results than the S3D_FES simulation, but with a level of error that is slightly above the S3D_Tugo simulation. As a reminder, T-UGOm was specifically designed to model and study tides, unlike SYMPHONIE, which is dedicated to circulation simulations. In this sense, it is logical that the performance of the Tugo forcing would be higher, although the results obtained with S2D are already an improvement from using the FES2012 atlas.

As an attempt to evaluate the impact of the tidal forcing on the hydrology and residual dynamics inside the domain, we have compared the simulations to the observations at our disposal. We found a significant impact on the representation of SSS at large scale $\left(1^{\circ} \times 1^{\circ}\right)$ and for monthly averages over the shelf. Possible mechanisms include the advection by surface current and the vertical mixing which is strongly influenced by tides over the shelf. On the other hand, comparisons to T, S profiles from single point measurements were inconclusive; this is not surprising as modeldata misfits at single points comprehend many possible sources of error, such as co-localization, that dominates the influence of the tidal solutions on stratification. Similarly, SSH time series comparisons showed very little differences in terms of RMSE or correlation, but the STD of the observed signal was better reproduced by S3D_Tugo ; this suggests that the SSH variability at the coast is better represented in this simulation. Finally, the daily 2D means of 

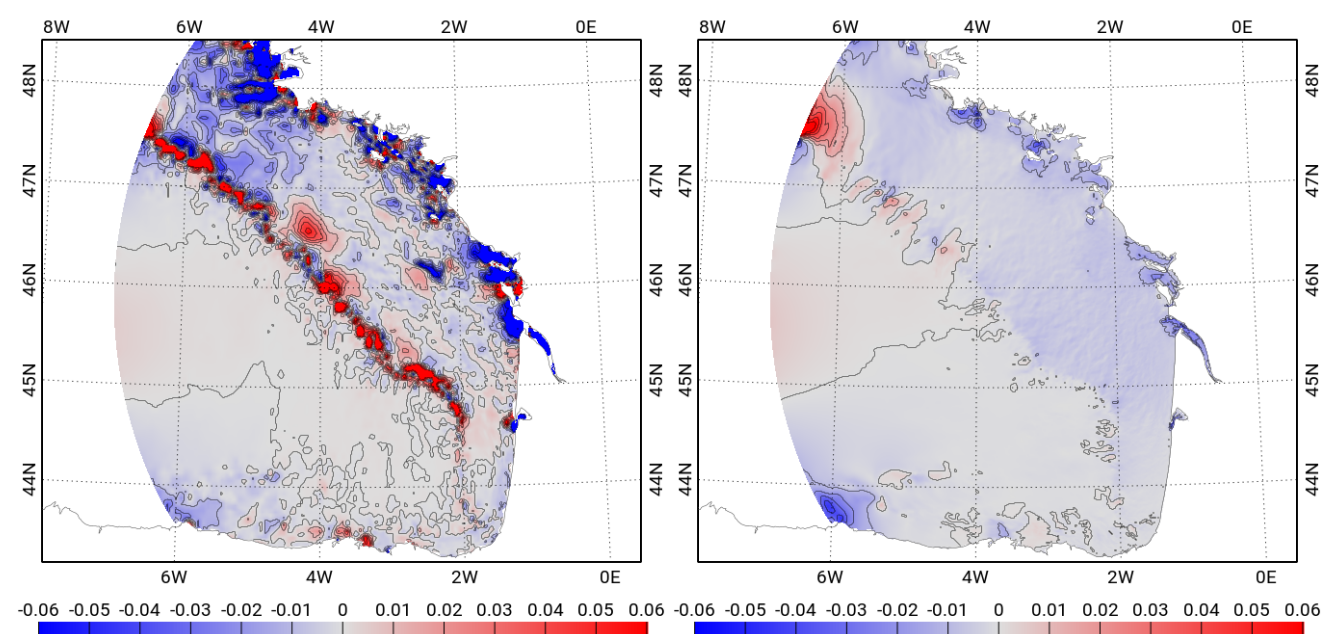

(a)

(b)

Figure 11: Difference between the amplitude of the 2D M2 current in: (a) FES2012 and Tugo, (b) S3D_FES and S3D_Tugo (m/s)

the along-shore current velocities from the ASPEX campaign time series are better correlated to the S3D_Tugo field than to the S3D_FES field; we evidenced episodes of a strong improvement of the model slope current in S3D_Tugo with respect to the data. An attempt is made to provide an interpretation to this improvement in the next section.

\subsection{Tidal forcing influence on the circulation}

In section 4.4, 2D daily currents obtained from ADCP data are compared to modelled currents. These comparisons clearly show a better agreement with the S3D_Tugo simulation, even though the tidal ellipses calculated at the same location (section 4.2) are very close between the three simulations. This suggests that, at these locations (far from the open boundaries), the tidal forcing seems to have a greater influence on the global circulation than on the tidal currents themselves. The difference between the S3D_FES and S3D_Tugo M2 tidal current (Figure 11b) confirms this observation: on the shelf and slope in the southeastern part of the Bay, the differences are very small compared to the large differences observed at the open boundaries.

To explore further the impact on the mean circulation, we compare the mean currents over ten days, from daily detided fields. Figure 12 shows the results obtained at a 50m depth, in the southern part of the domain, for the S3D_FES and S3D_Tugo simulations, over the first 10 days of January. This period has been chosen because it corresponds to the one where the differences at the ASPEX 9 mooring are the largest (Figure 8). It also coincides with the occurrence of an eastward along slope current with an amplitude locally larger than $25 \mathrm{~cm} / \mathrm{s}$. The associated SST field displays a warm water tongue along the Spanish coast that extends northward along the slope up to $45^{\circ} \mathrm{N}$ off the French coast. This SST patterns is consistent with the classical view in the literature of a poleward slope current in early winter advecting warm water masses (see for instance Pingree and Le Cann (1992)). On Figure 12, differences between the simulations, at small scales in the mesoscale field, for both current and SST, can be interpreted as a 

and $4^{\circ} \mathrm{W}$, and $3^{\circ} \mathrm{W}$ and $2^{\circ} \mathrm{W}$.
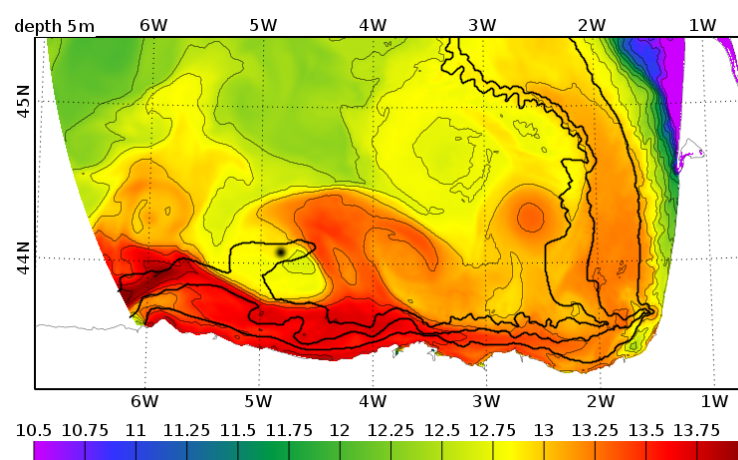

(a)

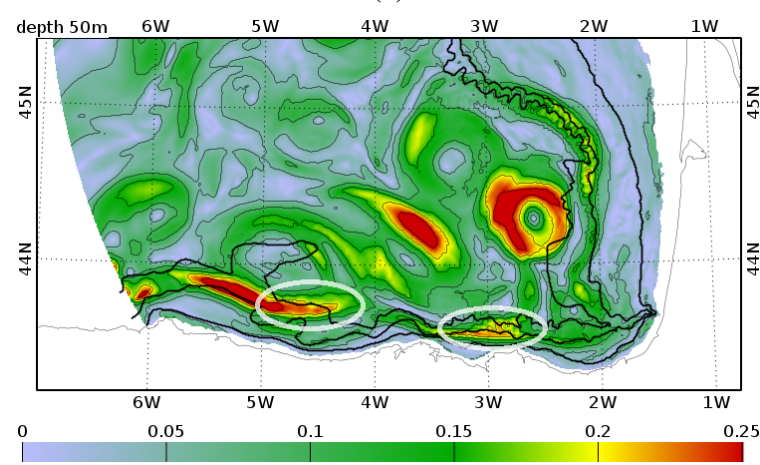

(c)

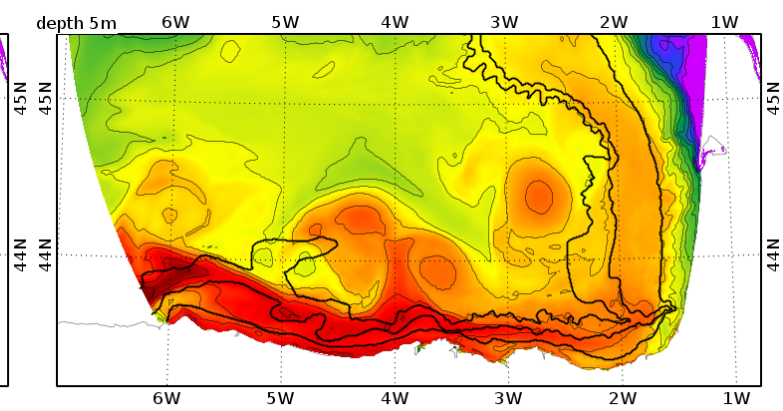

(b)

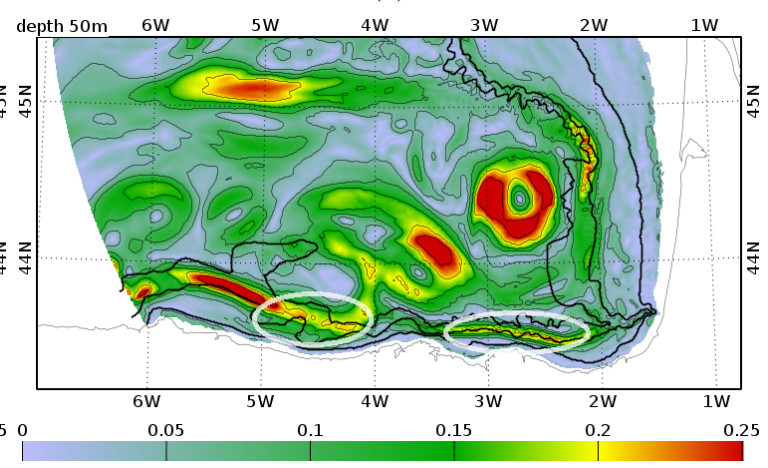

(d)

Figure 12: Comparison between 10-day means (January 1 to 10$)$ of sea surface temperature $\left({ }^{\circ} \mathrm{C}\right)$ and current velocities (m/s) at 50m from S3D_FES (a,c) and S3D_Tugo (b,d). The Le Danois Bank is marked with a black dot in (a). In black: 100, 200 and 1000m isobaths.

These different features are observed just east from the Le Danois Bank (approximately at $44^{\circ} 05^{\prime} \mathrm{N}, 4^{\circ} 50^{\prime} \mathrm{W}$ ), which is a seamount-like topographic feature at about $60 \mathrm{~km}$ of the northern Spanish coast (González-Pola et al., 2012), quite close to the open boundaries of our configuration. From in-situ measurements, González-Pola et al. (2012) show that diurnal tides (K1 and O1) are strongly amplified, both on the northern and southern sides of this seamount. The authors interpret the amplification of the diurnal tides as resulting from the generation of resonant seamount trapped waves. They also argue that the amplification of K1, O1, and, to a lesser extent, of M2, could be an indicator of topographic tidal rectification, that can generate a mean residual current.

Tidal rectification occurs when the nonlinear terms in the momentum equation become of the order or greater than other forcing terms; the processes involved are a combination of continuity and Coriolis effects and bottom friction (Loder, 1980). Shelf breaks or seamounts in macrotidal environments, with a strong cross-isobath tidal flow, are 
favourable sites for the occurrence of topographic tidal rectification. For instance, Garreau and Maze (1992) derive analytical solutions for eulerian currents generated by the rectification of the M2 tides over a slope: they find that the solution is consistent with observed residual current at the top of the shelf break in the northern Bay of Biscay.

The Le Danois Bank western and northern slopes are a priori favorably oriented so that M2 tides can indeed rectify. Based on rough scaling arguments, González-Pola et al. (2012)show that M2 rectified flow could reach there a few centimeters per second.

In our simulation, because of the multiple forcing terms that are resolved by our model, it is difficult to isolate tidal rectification processes (a thorough analysis of tidal rectification in our simulations is beyond the scope of this study). But in view of the studies in the literature and of the general topographic characteristics, we find very likely that topographic tidal rectification indeed occurs in our runs in the Le Danois area. In our simulations, the amplification of K1 and $\mathrm{O} 1$ does occur, with a stronger intensity in S3D_Tugo. Because of the proximity to the open boundary, the tidal current shows significant differences there between the S3D_FES et S3D_Tugo runs (Figure 11). We therefore expect the rectified flow to be different as well. The early January period is characterized by a mean along-slope current, at least partially originated outside of our domain. We suggest that tidal-mean current interactions and topographic rectification mechanisms impact the along-slope mean flow; the observed difference further downstream, at the location of the ASPEX 9 mooring (Figure 8) would then result from this impact. We also performed a simulation without tides (not shown), and found that the 10-day mean currents and SST were significantly modified in this part of the Bay, thus confirming the strong impact of tides on circulation.

Tidal rectification appears as a likely propagator within the domain of the differences on tidal currents observed at the open boundaries (Figure 11).

\subsection{Combining tidal forcing and OGCM forcing: potential impact of imperfectly detided fields?}

In this section, we open the discussion to another issue, which is not directly related to the two previous ones but that remains central in the general problem of downscaling tides.

In an attempt to downscale in a regional (child) model both the tidal and non-tidal (i.e. general circulation) dynamics, one faces two possible strategies: 1/ chose a parent model that simulates both tides and the general circulation and use the total parents fields (tidal plus non-tidal), 2/ use separate boundary conditions for the tidal and non-tidal dynamics. The first strategy requires that the tidal signal prescribed at the open boundaries and the one generated inside the domain are in phase which is very likely not to happen. Reasons for this situation not to happen are, as illustrated in this study and commented in section 5.2, the usual inconsistency between the bathymetry and the difference of resolution between the parent and child models. Other possible causes include the difference of parameterizations (for instance for the bottom friction) and other forcings. This is the reason why the coastal modelling communities (MacCready et al., 2009; Dong et al., 2011; Katavouta and Thompson, 2016) usually consider separately the openboundary forcing fields for tides and for the general circulation. This raises however another issue that we address below. 
Indeed, in case the second strategy is chosen, the forcing fields for the general circulation (from the parent model) must be completely detided. If they are imperfectly detided, the residual tidal signal may be aliased and impact the interior solution. In our case, the parent fields from the IBI/NEMO model are provided every $24 \mathrm{~h}$ as averages over 25 hours. Averaging over 25 hours is a very efficient way to remove the M2 signal (period of $12.4 \mathrm{hr}$ ) which is by far the largest constituent in the Bay of Biscay. It also partly removes the other semi-diurnal and diurnal components. A thorough look at the effect on S2 tides however shows a significant residual at the MSf frequency (i.e. at the fortnightly period of the spring-neap cycle). In the IBI solutions, we find a residual signal of up to $3 \mathrm{~cm}$ in the northern part of the domain, and approximately $1 \mathrm{~cm}$ on average in the whole domain (Figure 13a). For comparison, the same tidal analysis has been performed on other daily fields from the MERCATOR-Ocean operational system with the NEMO model running without tides: the so-called PSY2V4R4 product with a 1/12 ${ }^{\circ}$ horizontal resolution and data assimilation. The analysis results in a much weaker signal at the MSf frequency, of less than $1 \mathrm{~cm}$ in the whole domain (and likely due to the assimilation of a residual MSf signal in the altimetric data) (Figure 13b).

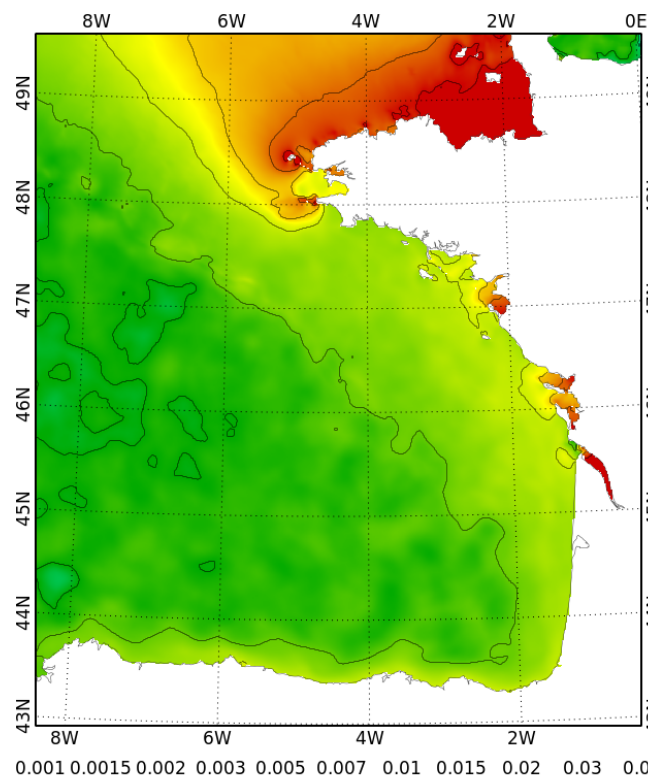

(a) IBI

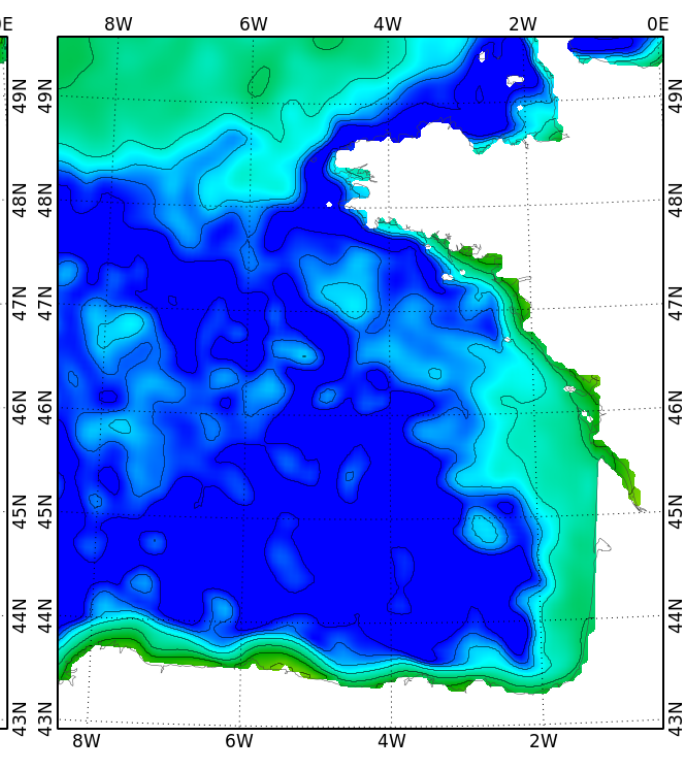

Figure 13: MSf residual (m) after a tidal analysis of daily fields of SSH

A 3D simulation forced by PSYV4R4 was performed to compare the results, in terms of tidal elevations, to the simulation forced by the IBI operational product. When compared to the tidal gauges (same dataset as the one used in section 3.4), the differences in terms of model-data misfits between the two runs are small on average for M2, S2 and M4 (0.1 to $0.2 \mathrm{~cm}$ in complex error). However, differences can be quite high locally, especially in, or close to the Gironde estuary for the M4 constituent. At the Royan and Port-Bloc and Richard tidal gauges, the complex errors are reduced by $2.2,1.9$ and $4.8 \mathrm{~cm}$ respectively. On the other hand, the errors are increased by $6.3,6.1$ and $6.1 \mathrm{~cm}$ 
respectively for the three following upstream stations (Lamena, Trompeloup and Fort-Médoc). Thus, although the overall error budget remains almost unchanged, the influence of the global forcing can have a strong influence locally.

In conclusion, the use of imperfectly detided 3D fields from the parent model leads here to a residual signal in $\mathrm{SSH}$ at the neap-spring frequency all over the domain $(1.1 \mathrm{~cm}$ on average when forced by IBI, $0.42 \mathrm{~cm}$ when forced by PSY2V4R4 (not shown)), and enhanced over the shelf. The impact on the tidal signal itself is weak on average except locally. Our comparison to tidal gauges shows that, in spite of this residual signal, there is no evidence of any degradation on the tides representation in the child model.

\section{Conclusion}

In regional and coastal modelling, a common way to handle open-boundary conditions consists in using external forcing fields from a model at basin scale in one-way nesting approach. Differences between parent and child models bathymetry and resolution mainly lead to inconsistencies between the parent forcing and the child dynamics (i.e. over-specification error as defined for instance by Herzfeld and Gillibrand (2015)). In this study, we propose a robust and simple approach to improve the downscaling of barotropic tides in a 3D circulation model. We have chosen to develop an approach that reduces the inconsistencies between the external fields and the interior solution. We do not try to improve the OBC scheme itself because we aim at proposing a generic approach that can work in many different configurations with different OBC equations and numerical implementations. By pre-processing the FES2012 tidal atlas with a 2D simulation (S2D or Tugo), we produce tidal fields generated with the same bathymetry and on the same grid as the 3D model.

Another solution could be to add a few cells in the 2D pre-processing configurations, in order to prevent the child model (3D) from inheriting any possible errors from the 2D model due to the open-boundary scheme (e.g. rim currents). This is not the option chosen here as we aimed to avoid defining and handling an extra configuration (the coastal domain of interest with extra cells). We have checked in the $2 \mathrm{D}$ solutions that there was indeed no evidence of reflection for M2 currents (which are the dominant tidal currents).

Results show the potential benefit of using a tailored tidal forcing to force a 3D circulation model. The generation of a $2 \mathrm{D}$ tidal solution on the same grid and bathymetry as the ones used in the 3D model reduces the errors due to interpolation and bathymetry inconsistencies at the open boundaries, especially on the tidal currents. Compared to the classical approach, that consists of using a tidal atlas, the use of the tidal model T-UGOm brings about a clear improvement in the tidal solution of the 3D simulation. This approach and method can be transposed to other 3D circulation models, particularly in areas where the tides play a key role on coastal dynamics.

Thanks to several datasets, the influence of the tidal forcing on the tidal solution and the circulation in a 3D circulation model was studied. The results show the benefit of considering both single-point data (tidal harmonics from tidal gauges, SSH time series, current velocities and tidal currents from ADCP data) and wide spatial coverage data (satellite altimetry tidal harmonics) to determine the best forcing. These findings underline the importance of using 
several datasets and diagnostics to validate a numerical model. Even though this configuration was conceived to study fine scale processes, the validation of the large scale circulation is crucial. This study particularly emphasizes the benefit of satellite altimetry, which provides regular time series homogeneous in space. Tidal gauges, although valuable, can exhibit significant differences in tidal elevations within a few kilometers, due to differences in instrumentation or the time series lengths for example.

Moreover, regional circulation model are often designed to reproduce and study small scale dynamics that occur very close to the coast. In shallow waters, the propagation and the distortion of the tide are strongly influenced by the topography and by bottom friction. For example, nonlinear interactions occurring between the tide and the topography result in the generation and/or amplification of overtides such as M4. Bottom friction strongly impacts the propagation of M2. To reproduce this behavior in numerical models, several tests are often required to tune the bottom friction. With 3D circulation models, this calibration can take a lot of time, both in terms of CPU and running time. With T-UGOm, a large number of tests can be performed in a day, because the running time is of the order of minutes, against hours or days for the 3D simulations. Even if we use the S2D approach presented in this study, the running time is still significantly higher than with T-UGOm in frequency-mode. Although the friction formulations are obviously different between models, the bathymetry and grid being exactly the same, a first approximation can easily be obtained, before adapting this tuning to the circulation model.

\section{Acknowledgments}

This study is part of the COCTO project (SWOT Science Team Program) funded by CNES. The authors wish to gratefully acknowledge the CNES for the funding of this work through a post-doctoral grant. This work is also a contribution to the LEFE/GMMC project ENIGME. HPC resources come from CALMIP (grants 2016 and 2017p1119) and GENCI (CINES, projects EGO7298 and EGO0098): support from CALMIP and GENCI is acknowledged.

The authors especially thank the $\mathrm{CTOH} / \mathrm{LEGOS}$ for providing the satellite altimetry data necessary to this study (http://ctoh.legos.obs-mip.fr/). Many thanks to Puertos del Estado, the SPC Gironde, REFMAR Météo-France and IFREMER (CORA-IBI database, Islands network), SHOM and OHI for providing the data. The authors gratefully acknowledge the receipt of SSS gridded data from E. Kestenare (LEGOS). G. Charria, S. Theetten and A. Le Boyer (IFREMER) are also particularly acknowledged for providing the ASPEX data and processing. We also thank C. Nguyen and T. Duhaut for their valuable help with the SYMPHONIE setting. Maps and model-data comparisons for tidal elevations have been made using POCViP, developed at LEGOS by D. Allain and F. Lyard.

\section{Appendix: Open-boundary conditions (OBC) in SYMPHONIE}

The numerical schemes used at the OB in Symphonie are described in Marsaleix et al. (2006) and are summarized in this appendix. 
The state variables of Symphonie are: the horizontal $(u, v)$ and vertical $(w)$ currents, temperature and salinity $(T, S)$ and the free surface elevation anomaly $(\eta)$ with respect to the state at rest. Other model variables are those used in the turbulence closure scheme and are not considered here. Barotropic $(\bar{u}, \bar{v})$ and baroclinic $\left(u^{\prime}, v^{\prime}\right)$ components of the current $\left(u=\bar{u}+u^{\prime}, v=\bar{v}+v^{\prime}\right)$ are computed separately using the time-splitting technique described by Blumberg and Mellor (1987). The barotropic velocities are computed as the depth-averaged velocities. The OBC are based on distinct formulations for the different variables and on the use of external forcing fields along the open boundaries.

\section{Barotropic variables}

For the barotropic variables, the OBC consist in:

- C1- a Flather condition applied to the free surface elevation anomaly $(\eta)$ and taking into account the external forcing

- C2- a radiative condition for the tangential component of the transport

- $\mathrm{C} 3$ - the transport component normal to the boundary is deduced from the continuity equation, from $\eta(\mathrm{C} 1)$ and from the tangential transport $(\mathrm{C} 2)$

Let us take the example of a domain with a western open boundary. Figure 14 shows the discretization and the location of the open boundary. Conditions $\mathrm{C} 1-\mathrm{C} 3$ write as follows at time t:

$$
\eta_{1, j}=\eta_{1, j}^{f}+\frac{1}{\sqrt{g H}}\left(U_{2, j}-U_{2, j}^{f}\right)
$$

$$
\left[\frac{\partial V}{\partial x}\right]_{2, j}=\left[\frac{\partial V^{f}}{\partial x}\right]_{2, j} \Rightarrow V_{1, j}-V_{1, j}^{f}=V_{2, j}-V_{2, j}^{f}
$$

$$
\frac{\partial \eta}{\partial t}+\frac{\partial U}{\partial x}+\frac{\partial V}{\partial y}=0 \Rightarrow U_{1, j}=U_{2, j}-\frac{d x}{d y}\left[V_{1, j+1}-V_{1, j}\right]+d x \frac{\partial \eta_{1, j}}{\partial t}
$$

where $\left(\eta^{f}, U^{f}, V^{f}\right)$ are the external forcing fields, $(U=(H+\eta) \bar{u}, V=(H+\eta) \bar{v})$ are the barotropic transports, $H$ the bathymetry and $g$ gravity.

Besides, the barotropic velocity is relaxed towards the external forcing within a sponge-layer in order to reduce possible reflection of the outgoing flow. There is no additional constraint on the global mass conservation. Indeed, Marsaleix et al. (2006) show that the mean sea surface elevation over the domain tends toward the mean sea surface elevation of the forcing field over a time scale of about $S / L c$, where $S$ is the area of the domain, $L$ the length of the open boundaries and $c$ the mean barotropic phase speed. Taking rough values for our domain $(L=500 \mathrm{~km}, H=$ $5000 m, S=L x L, c=\sqrt{g H}$ ) we obtain a time scale of 0.6 hours.

The reason for the effectiveness of the Flather condition is discussed in Blayo and Debreu (2005). Besides, we note that with such a choice of conditions and of implementation on the Arakawa $\mathrm{C}$ grid (Figure 14), for any point inside the domain, the first order (linear) terms in the equation of motions depend on the elevation and tangential velocity at the boundary only. The normal velocity at the boundary enters the second-order advection and diffusion terms. As 


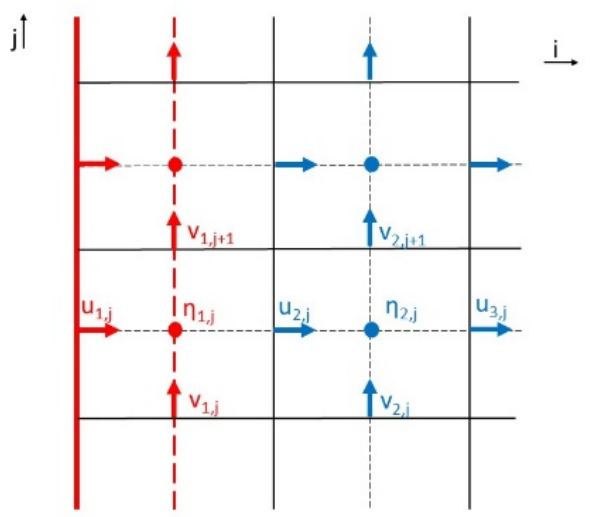

Figure 14: Implementation of the OBC for a western boundary on the SYMPHONIE grid. The boundary points are shown with red symbols, while interior points are shown in blue.

noted by Herzfeld and Andrewartha (2012), this allows to minimize the impact of uncertainties in the normal velocity, which is critical since the normal velocity is responsible for import/export of mass and energy through the boundary.

\section{Baroclinic variables}

For the baroclinic variables, a radiation condition is applied to the perturbations of $\left(u^{\prime}, v^{\prime}\right)$ from the external forcing as explained by Marsaleix et al. (2006) (see their equation 28). $\left(u^{\prime}, v^{\prime}\right)$ are also relaxed toward the external fields in a sponge-layer. The overall condition writes:

C4- $B u^{\prime}=B u^{f}$ and $B v^{\prime}=B v^{f}$ where the operator $B$ is given by $B=\frac{\partial \dot{\partial}}{\partial t}+c \frac{\partial \cdot}{\partial n}-\dot{\bar{\tau}}$

Condition $\mathrm{C} 4$ ensures that the child solution and the external forcing give the same response to the boundary operator $B$, which is a way of imposing consistency, as recommended by Blayo and Debreu (2005). The speed c is constant and of an order of magnitude comparable to that of the phase speed of the internal waves (here $c=1 \mathrm{~m} / \mathrm{s}$ ).

In the BOBSHELF configuration, the sponge-layer is 30 points wide (about $60 \mathrm{~km}$ considering the horizontal resolution near the open boundaries of the domain) and $\tau$ decreases from 1 day at the closest point to the openboundary to one hundredth of its value at the 30th point inside the domain.

\section{Temperature and salinity}

The temperature and salinity conservation equations are used to compute the boundary conditions in $T, S$, as justified by Marsaleix et al. (2006). These involve the velocities at the OB as computed from conditions C1-C4. The upwind advection scheme adjusts its calculation according to the sign of the current component that is normal to the open boundary. In incoming conditions, temperature and salinity open boundary fluxes are calculated using the external $T, S$ fields and, in the opposite case, considering the $T, S$ fields of the interior solution.

\section{External forcing}


The external forcing comes from a tidal model (or tidal atlas) and from a general circulation model (parent model) for the residual circulation, as explained in section 3.1. To be more precise:

- $\eta^{f}$ is the sum of the elevations for the nine tidal constituents provided by the tidal atlas and of the non-tidal sea level elevation provided by the parent circulation model ;

- $\left(U^{f}, V^{f}\right)$ are the sum of the horizontal transport for the nine tidal constituents and of the horizontal non-tidal transport from the parent model ;

- the external forcing fields for $\left(u^{\prime}, v^{\prime}, T, S\right)$ are given by the parent model.

\section{Dirichlet (clamped) condition used for the 2D SYMPHONIE simulation}

In the 2D SYMPHONIE run (S2D) performed to generate the tidal forcing (see section 3.3), the Flather condition $\mathrm{C} 1$ is replaced by a Dirichlet condition that writes:

$\eta=\eta^{f}$

In this case, $\eta^{f}$ is the tidal elevation given by FES2012, since for the 2D run we exclude any other forcing than tides (no atmospheric forcing field, no river runoff, no residual circulation at the open boundaries). Conditions $\mathrm{C} 2-\mathrm{C} 4$ are unchanged and the barotropic velocity is relaxed towards the external current (here from FES2012) in the sponge layer as described above; the relaxation is expected to reduce possible reflection in case of outgoing conditions, while the absence of any other forcing than tides limits the generation of additional waves propagating towards the open boundary. We have checked that the main tidal current (M2 constituent) does not indeed show any spurious patterns close to the open boundary.

\section{References}

Alory, G., Delcroix, T., Téchiné, P., Diverrès, D., Varillon, D., Cravatte, S., Gouriou, Y., Grelet, J., Jacquin, S., Kestenare, E., Maes, C., Morrow, R., Perrier, J., Reverdin, G., Roubaud, F., 2015. The French contribution to the voluntary observing ships network of sea surface salinity. Deep-Sea Research Part I: Oceanographic Research Papers 105, 1-18. doi:10.1016/j.dsr.2015.08.005.

Birol, F., Fuller, N., Lyard, F., Cancet, M., Niño, F., Delebecque, C., Fleury, S., Toublanc, F., Melet, A., Saraceno, M., Léger, F., 2016. Coastal applications from nadir altimetry: example of the X-TRACK regional products. Advances in Space Research 59, 936-953. doi:10.1016/j.asr.2016.11.005.

Blayo, E., Debreu, L., 2005. Revisiting open boundary conditions from the point of view of characteristic variables. Ocean Modelling 9, 231-252. doi:10.1016/j.ocemod.2004.07.001.

Blumberg, A.F., Mellor, G.L., 1987. A description of a three-dimensional coastal ocean circulation model. Three-dimensional coastal ocean models , $1-16$.

Carrère, L., Lyard, F., Cancet, M., Guillot, A., Roblou, L., 2012. FES2012: a new global tidal model taking advantage of nearly 20 years of altimetry, in: Proceedings of 20 years of Altimetry, Venice.

Carter, G.S., Merrifield, M.a., 2007. Open boundary conditions for regional tidal simulations. Ocean Modelling 18, 194-209. doi:10.1016/j.ocemod.2007.04.003.

Cartwright, D., Edden, A.C., Spencer, R., Vassie, J., 1980. The tides of the northeast Atlantic Ocean. Philosophical Transactions of the Royal Society of London A 298, 87-139. 
Cavanie, A., Hyacinthe, J., 1976. Etude des courants et de la marée à la limite du plateau continental d'après les mesures effectuées pendant la campagne Golfe de Gascogne 1970 . Technical Report. Ifremer.

Damien, P., Bosse, A., Testor, P., Marsaleix, P., Estournel, C., 2017. Modeling postconvective submesoscale coherent vortices in the northwestern Mediterranean Sea. Journal of Geophical Research doi:10.1002/2014JC010094.

Dong, C., McWilliams, J.C., Hall, A., Hughes, M., 2011. Numerical simulation of a synoptic event in the Southern California Bight. Journal of Geophysical Research 116. doi:10.1029/2010JC006578.

Ebert, E.E., 2008. Fuzzy verification of high-resolution gridded forecasts: a review and proposed framework. Meteorological applications 15, $51-64$.

Egbert, G.D., Erofeeva, S.Y., 2002. Efficient inverse modeling of barotropic ocean tides. Journal of Atmospheric and Oceanic Technology 19, 183-204. doi:10.1175/1520-0426(2002)019<0183:eimobo >2.0.co;2.

Fu, L.L., Alsdorf, D., Morrow, R., Rodriguez, E., Mognard, N. (Eds.), 2012. SWOT: The Surface Water and Ocean Topography Mission: Wide-Swath Altimetric Measurement of Water Elevation on Earth. Jet Propulsion Laboratory, Pasadena, California. doi:10.1017/CBO9781107415324.004.

Garreau, P., Maze, R., 1992. Tidal rectification and mass transport over a shelf break: a barotropic frictionless model. Journal of Physical Oceanography 22, 719-731.

González-Pola, C., Díaz del Río, G., Ruiz-Villarreal, M., Sánchez, R.F., Mohn, C., 2012. Circulation patterns at Le Danois Bank, an elongated shelfadjacent seamount in the Bay of Biscay. Deep-Sea Research Part I: Oceanographic Research Papers 60, 7-21. doi:10.1016/j.dsr.2011.10.001.

Guarnieri, A., Pinardi, N., Oddo, P., Bortoluzzi, G., Ravaioli, M., 2013. Impact of tides in a baroclinic circulation model of the Adriatic Sea. Journal of Geophysical Research: Oceans 118, 166-183. doi:10.1029/2012JC007921.

Herzfeld, M., 2009. Improving stability of regional numerical ocean models. Ocean Dynamics 59, 21-46. doi:10.1007/s10236-008-0158-1.

Herzfeld, M., Andrewartha, J., 2012. A simple, stable and accurate dirichlet open boundary condition for ocean model downscaling. Ocean Modelling 43, 1-21.

Herzfeld, M., Gillibrand, P.a., 2015. Active open boundary forcing using dual relaxation time-scales in downscaled ocean models. Ocean Modelling 89, 71-83. doi:10.1016/j.ocemod.2015.02.004.

Holt, J., Hyder, P., Ashworth, M., Harle, J., Hewitt, H.T., Liu, H., New, A.L., Pickles, S., Porter, A., Popova, E., Allen, J.I., Siddorn, J., Wood, R., 2017. Prospects for improving the representation of coastal and shelf seas in global ocean models. Geoscientific Model Development 10, 499-523. doi:10.5194/gmd-2016-145.

Katavouta, A., Thompson, K.R., 2016. Downscaling ocean conditions with application to the Gulf of Maine, Scotian Shelf and adjacent deep ocean. Ocean Modelling 104, 54-72. doi:10.1016/j.ocemod.2016.05.007.

Kersalé, M., Marié, L., Le Cann, B., Serpette, A., Lathuilière, C., Le Boyer, A., Rubio, A., Lazure, P., 2016. Poleward along-shore current pulses on the inner shelf of the Bay of Biscay. Estuarine, Coastal and Shelf Science 179, 155-171. doi:10.1016/j.ecss.2015.11.018.

Large, W.G., Yeager, S.G., 2004. Diurnal to decadal global forcing for ocean and sea-ice models: The data sets and flux climatologies. NCAR Tech. Note TN-460+ST. doi:10.5065/D6KK98Q6.

Le Boyer, A., Charria, G., Le Cann, B., Lazure, P., Marié, L., 2013. Circulation on the shelf and the upper slope of the Bay of Biscay. Continental Shelf Research 55, 97-107. doi:10.1016/j.csr.2013.01.006.

Le Cann, B., 1990. Barotropic tidal dynamics of the Bay of Biscay shelf. Continental Shelf Research 10, 723-758.

Loder, J.W., 1980. Topographic Rectification of Tidal Currents on the Sides of Georges Bank. Journal of Physical Oceanography 10, $1399-1416$. doi:10.1175/1520-0485(1980)010<1399:TROTCO >2.0.CO;2.

Lyard, F., Lefevre, F., Letellier, T., Francis, O., 2006. Modelling the global ocean tides: Modern insights from FES2004. Ocean Dynamics 56, 394-415. doi:10.1007/s10236-006-0086-X.

MacCready, P., Banas, N.S., Hickey, B.M., Dever, E.P., Liu, Y., 2009. A model study of tide- and wind-induced mixing in the Columbia River Estuary and plume. Continental Shelf Research 29, 278-291. doi:10.1016/j.csr.2008.03.015.

Madec, G., 2008. NEMO Ocean General Circulation Model Reference Manual. Internal Report LODYC/IPSL. 
Maraldi, C., Chanut, J., Levier, B., Ayoub, N., De Mey, P., Reffray, G., Lyard, F., Cailleau, S., Drévillon, M., Fanjul, E.a., Sotillo, M.G., Marsaleix, P., 2013. NEMO on the shelf: Assessment of the Iberia-Biscay-Ireland configuration. Ocean Science 9, 745-771. doi:10.5194/os-9-745-2013.

Marsaleix, P., Auclair, F., Duhaut, T., Estournel, C., Nguyen, C., Ulses, C., 2012. Alternatives to the Robert-Asselin filter. Ocean Modelling 41, 53-66. doi:10.1016/j.ocemod.2011.11.002.

Marsaleix, P., Auclair, F., Estournel, C., 2006. Considerations on Open Boundary Conditions for Regional and Coastal Ocean Models. Journal of Atmospheric and Oceanic Technology 23, 1604-1613. doi:10.1175/JTECH1930.1.

Marsaleix, P., Auclair, F., Floor, J.W., Herrmann, M.J., Estournel, C., Pairaud, I., Ulses, C., 2008. Energy conservation issues in sigma-coordinate free-surface ocean models. Ocean Modelling 20,61-89. doi:10.1016/j.ocemod.2007.07.005.

Marsaleix, P., Ulses, C., Pairaud, I., Herrmann, M.J., Floor, J.W., Estournel, C., Auclair, F., 2009. Open boundary conditions for internal gravity wave modelling using polarization relations. Ocean Modelling 29, 27-42. doi:10.1016/j.ocemod.2009.02.010.

Michaud, H., Marsaleix, P., Leredde, Y., Estournel, C., Bourrin, F., Lyard, F., Mayet, C., Ardhuin, F., 2012. Three-dimensional modelling of wave-induced current from the surf zone to the inner shelf. Ocean Science 8, 657-681. doi:10.5194/os-8-657-2012.

Neumann, L.E., ŠimÅnek, J., Cook, F.J., 2011. Implementation of quadratic upstream interpolation schemes for solute transport into HYDRUS-1D. Environmental Modelling and Software 26, 1298-1308. doi:10.1016/j.envsoft.2011.05.010.

Oliger, J., Sundström, A., 1978. Theoretical and practical aspects of some initial boundary value problems in fluid dynamics. SIAM Journal on Applied Mathematics 35, 419-446.

Orain, F., 2016. Product user manual for Level 3 SST products over European Seas. Technical Report. CMEMS.

Pairaud, I.L., Auclair, F., Marsaleix, P., Lyard, F., Pichon, A., 2010. Dynamics of the semi-diurnal and quarter-diurnal internal tides in the Bay of Biscay. Part 2: Baroclinic tides. Continental Shelf Research 30, 253-269. doi:10.1016/j.csr.2009.10.008.

Pairaud, I.L., Lyard, F., Auclair, F., Letellier, T., Marsaleix, P., 2008. Dynamics of the semi-diurnal and quarter-diurnal internal tides in the Bay of Biscay. Part 1: Barotropic tides. Continental Shelf Research 28, 1294-1315. doi:10.1016/j.csr.2008.03.004.

Pingree, R.D., Le Cann, B., 1992. Three anticyclonic slope water oceanic eDDIES (SWODDIES) in the Southern Bay of Biscay in 1990. Deep Sea Research Part A, Oceanographic Research Papers 39, 1147-1175. doi:10.1016/0198-0149(92)90062-X.

Ray, R.D., 1999. A Global Ocean Tide Model From TOPEX/POSEIDON Altimetry: GOT99.2. Technical Report. NASA Tech. Memo. 209478. Goddard Space Flight Center, Greenbelt, MD. doi:1999-209478.

Reverdin, G., Kestenare, E., Frankignoul, C., Delcroix, T., 2007. Surface salinity in the Atlantic Ocean (30S-50N). Progress in Oceanography 73, 311-340. doi:10.1016/j.pocean.2006.11.004.

Rodriguez, E., Fernandez, D.E., Peral, E., Chen, C.W., Bleser, J.w.D., Williams, B., 2017. Wide-Swath Altimetry: A Review, in: Satellite altimetry over oceans and land surfaces. Taylor and Francis (in press). chapter 2.

Sottolichio, A., Castaing, P., 1999. A synthesis on seasonal dynamics of highly-concentrated structures in the Gironde estuary. Comptes Rendus de l'Académie des Sciences 329, 795-800.

Stammer, D., Ray, R., Andersen, O., Arbic, B., Bosch, W., Carrère, L., Cheng, Y., Chinn, D., Dushaw, B., Egbert, G., Erofeeva, S., Fok, H., Green, J., Griffiths, S., King, M., Lapin, V., Lemoine, F., Luthcke, S., Lyard, F., Morison, J., Müller, M., Padman, L., Richman, J., Shriver, J., Shum, C., Taguchi, E., Yi, Y., 2014. Accuracy assessment of global barotropic ocean tide models. Reviews of Geophysics 52, $243-282$. doi:10.1002/2014RG000450.

Szekely, T., Bezaud, M., Pouliquen, S., Reverdin, G., Charria, G., 2017. CORA-IBI, Coriolis Ocean Dataset for Reanalysis for the Ireland-BiscayIberia region. SEANOE doi:http://doi.org/10.17882/50360.

Toublanc, F., Brenon, I., Coulombier, T., 2016. Formation and structure of the turbidity maximum in the macrotidal Charente estuary (France): Influence of fluvial and tidal forcing. Estuarine, Coastal and Shelf Science 169, 1-14. doi:10.1016/j.ecss.2015.11.019.

Wang, X., Chao, Y., Zhang, H., Farrara, J., Li, Z., Jin, X., Park, K., Colas, F., McWilliams, J.C., Paternostro, C., Shum, C.K., Yi, Y., Schoch, C., Olsson, P., 2013. Modeling tides and their influence on the circulation in Prince William Sound, Alaska. Continental Shelf Research 63, S126-S137. doi:10.1016/j.csr.2012.08.016.

Zheng, L., Weisberg, R.H., 2012. Modeling the west Florida coastal ocean by downscaling from the deep ocean, across the continental shelf and 
into the estuaries. Ocean Modelling 48, 10-29. doi:10.1016/j.ocemod.2012.02.002. 